\title{
Audio-visual presentation of information for informed consent for participation in clinical trials (Review)
}

\author{
Ryan R, Prictor M, McLaughlin KJ, Hill S
}

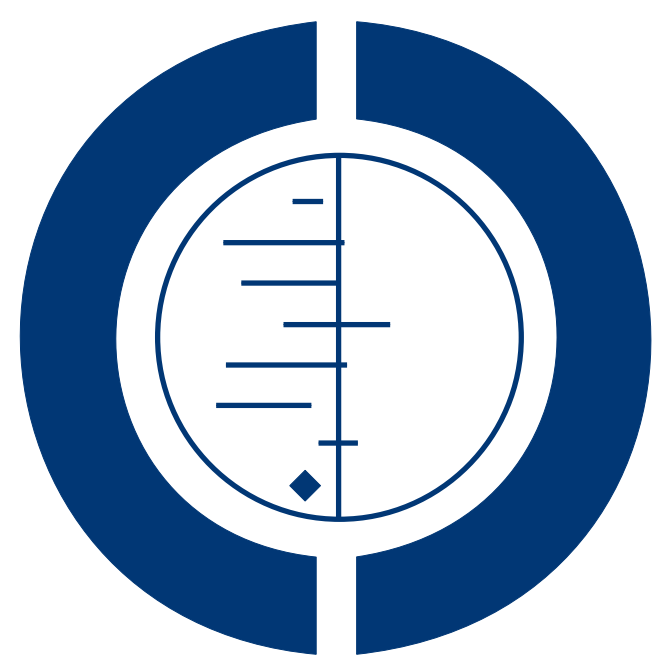

\section{THE COCHRANE COLLABORATION $^{\circledR}$}

This is a reprint of a Cochrane review, prepared and maintained by The Cochrane Collaboration and published in The Cochrane Library 2008, Issue 1

http://www.thecochranelibrary.com

\section{WILEY}

Audio-visual presentation of information for informed consent for participation in clinical trials (Review)

Copyright () 2009 The Cochrane Collaboration. Published by John Wiley \& Sons, Ltd. 
TABLE OF CONTENTS

HEADER . . . . . . . . . . . . . . . . . . . . . . . . . . . . . . . . . . . . 1

ABSTRACT . . . . . . . . . . . . . . . . . . . . . . . . . . . . . . . . . . . . . . . . . . . . . . .

PLAIN LANGUAGE SUMMARY . . . . . . . . . . . . . . . . . . . . . . . . . . . . . . . . . . . 2

BACKGROUND . . . . . . . . . . . . . . . . . . . . . . . . . . . . . . . . . . . . . . . . .

OBJECTIVES . . . . . . . . . . . . . . . . . . . . . . . . . . . . . . . . . . . . . . . . 44

METHODS . . . . . . . . . . . . . . . . . . . . . . . . . . . . . . . . . . . . . . 5

RESULTS . . . . . . . . . . . . . . . . . . . . . . . . . . . . . . . . . . . . . . . 7

DISCUSSION . . . . . . . . . . . . . . . . . . . . . . . . . . . . . . . . . . . . . . 13

AUTHORS' CONCLUSIONS . . . . . . . . . . . . . . . . . . . . . . . . . . . . . . . . 17

ACKNOWLEDGEMENTS . . . . . . . . . . . . . . . . . . . . . . . . . . . . . . . . .

REFERENCES . . . . . . . . . . . . . . . . . . . . . . . . . . . . . . . . . . . . . . 18

CHARACTERISTICS OF STUDIES . . . . . . . . . . . . . . . . . . . . . . . . . . . . . . . . . . . . . .

DATA AND ANALYSES . . . . . . . . . . . . . . . . . . . . . . . . . . . . . . . . . . . . . . . . . . . . . . . .

ADDITIONAL TABLES . . . . . . . . . . . . . . . . . . . . . . . . . . . . . . . . . . . . . . . . .

APPENDICES . . . . . . . . . . . . . . . . . . . . . . . . . . . . . . . . . . . . . . . . 45

WHAT'S NEW . . . . . . . . . . . . . . . . . . . . . . . . . . . . . . . . . . 53

HISTORY . . . . . . . . . . . . . . . . . . . . . . . . . . . . . . . . . . . . . . . 54

CONTRIBUTIONS OF AUTHORS . . . . . . . . . . . . . . . . . . . . . . . . . . . . . . . . . . 54

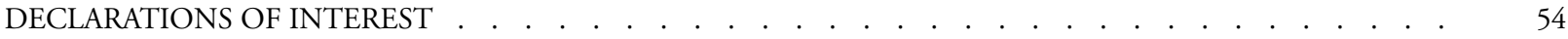

SOURCES OF SUPPORT . . . . . . . . . . . . . . . . . . . . . . . . . . . . . . . . . . . . . . . . . . .

INDEX TERMS . . . . . . . . . . . . . . . . . . . . . . . . . . . . . . . . . . . . 55

Audio-visual presentation of information for informed consent for participation in clinical trials (Review)

Copyright $\odot 2009$ The Cochrane Collaboration. Published by John Wiley \& Sons, Ltd. 


\title{
[Intervention Review]
}

\section{Audio-visual presentation of information for informed consent for participation in clinical trials}

\author{
Rebecca Ryan ${ }^{1}$, Megan Prictor $^{1}$, Kristin J McLaughlin ${ }^{2}$, Sophie Hill ${ }^{1}$ \\ ${ }^{1}$ Cochrane Consumers and Communication Review Group, Australian Institute for Primary Care, La Trobe University, Bundoora, \\ Australia. ${ }^{2} \mathrm{c}$ - Cochrane Consumers and Communication Review Group, Australian Institute for Primary Care, La Trobe University, \\ Bundoora, Australia
}

Contact address: Rebecca Ryan, Cochrane Consumers and Communication Review Group, Australian Institute for Primary Care, La Trobe University, Bundoora, VIC, 3086, Australia. r.ryan@latrobe.edu.au.

Editorial group: Cochrane Consumers and Communication Group.

Publication status and date: Edited (no change to conclusions), published in Issue 3, 2009.

Review content assessed as up-to-date: 7 November 2007.

Citation: Ryan R, Prictor M, McLaughlin KJ, Hill S. Audio-visual presentation of information for informed consent for participation in clinical trials. Cochrane Database of Systematic Reviews 2008, Issue 1. Art. No.: CD003717. DOI: 10.1002/14651858.CD003717.pub2.

Copyright (C) 2009 The Cochrane Collaboration. Published by John Wiley \& Sons, Ltd.

\begin{abstract}
A B S T R A C T

\section{Background}

Informed consent is a critical component of clinical research. Different methods of presenting information to potential participants of clinical trials may improve the informed consent process. Audio-visual interventions (presented for example on the Internet, DVD, or video cassette) are one such method.
\end{abstract}

\section{Objectives}

To assess the effects of providing audio-visual information alone, or in conjunction with standard forms of information provision, to potential clinical trial participants in the informed consent process, in terms of their satisfaction, understanding and recall of information about the study, level of anxiety and their decision whether or not to participate.

\section{Search methods}

We searched: the Cochrane Consumers and Communication Review Group Specialised Register (searched 20 June 2006); the Cochrane Central Register of Controlled Trials (CENTRAL), The Cochrane Library, issue 2, 2006; MEDLINE (Ovid) (1966 to June week 1 2006); EMBASE (Ovid) (1988 to 2006 week 24); and other databases. We also searched reference lists of included studies and relevant review articles, and contacted study authors and experts. There were no language restrictions.

\section{Selection criteria}

Randomised and quasi-randomised controlled trials comparing audio-visual information alone, or in conjunction with standard forms of information provision (such as written or oral information as usually employed in the particular service setting), with standard forms of information provision alone, in the informed consent process for clinical trials. Trials involved individuals or their guardians asked to participate in a real (not hypothetical) clinical study.

\section{Data collection and analysis}

Two authors independently assessed studies for inclusion and extracted data. Due to heterogeneity no meta-analysis was possible; we present the findings in a narrative review. 


\section{Main results}

We included 4 trials involving data from 511 people. Studies were set in the USA and Canada. Three were randomised controlled trials (RCTs) and the fourth a quasi-randomised trial. Their quality was mixed and results should be interpreted with caution.

Considerable uncertainty remains about the effects of audio-visual interventions, compared with standard forms of information provision (such as written or oral information normally used in the particular setting), for use in the process of obtaining informed consent for clinical trials. Audio-visual interventions did not consistently increase participants' levels of knowledge/understanding (assessed in four studies), although one study showed better retention of knowledge amongst intervention recipients. An audio-visual intervention may transiently increase people's willingness to participate in trials (one study), but this was not sustained at two to four weeks postintervention. Perceived worth of the trial did not appear to be influenced by an audio-visual intervention (one study), but another study suggested that the quality of information disclosed may be enhanced by an audio-visual intervention. Many relevant outcomes including harms were not measured. The heterogeneity in results may reflect the differences in intervention design, content and delivery, the populations studied and the diverse methods of outcome assessment in included studies.

\section{Authors' conclusions}

The value of audio-visual interventions for people considering participating in clinical trials remains unclear. Evidence is mixed as to whether audio-visual interventions enhance people's knowledge of the trial they are considering entering, and/or the health condition the trial is designed to address; one study showed improved retention of knowledge amongst intervention recipients. The intervention may also have small positive effects on the quality of information disclosed, and may increase willingness to participate in the shortterm; however the evidence is weak. There were no data for several primary outcomes, including harms. In the absence of clear results, triallists should continue to explore innovative methods of providing information to potential trial participants.

Further research should take the form of high-quality randomised controlled trials, with clear reporting of methods. Studies should conduct content assessment of audio-visual and other innovative interventions for people of differing levels of understanding and education; also for different age and cultural groups. Researchers should assess systematically the effects of different intervention components and delivery characteristics, and should involve consumers in intervention development. Studies should assess additional outcomes relevant to individuals' decisional capacity, using validated tools, including satisfaction; anxiety; and adherence to the subsequent trial protocol.

\section{PLAIN LANGUAGE SUMMARY}

\section{Audio-visual presentation of information used in the informed consent process for people considering entering clinical trials}

Informed consent is important for people who are thinking about participating in a clinical trial. Information for informed consent could be presented on the Internet, DVD, video cassette or by other means.

We conducted thorough searches for randomised and quasi-randomised controlled trials of information about trial participation that contained some audiovisual component compared with standard information (such as written or oral information as usually provided in the particular setting). We found four relevant studies, all set in the USA and Canada. The four studies varied in terms of the design and type of the audio-visual information, its content and delivery, the people participating in the informed consent study and the different ways of measuring outcomes. While study quality was mixed, three of the studies attempted to minimise at least some sources of potential bias.

Uncertainty remains about the effects of audio-visual information for informed consent, compared with standard forms of information provision, for people thinking about participating in a clinical trial. All four studies assessed knowledge and/or understanding of the trial to which people's informed consent was being sought. Audio-visual interventions did not consistently increase participants' levels of knowledge/understanding, although one study showed better retention of knowledge amongst intervention recipients. One study showed that an audio-visual intervention could briefly increase people's willingness to participate in trials, but this was not sustained two to four weeks post-intervention. The audio-visual intervention did not affect people's views of the worth of the trial they were considering joining (one study). Another study found that an audio-visual intervention may enhance the quality of the information conveyed to participants. Many outcomes including possible harms were not measured.

Audio-visual presentation of information for informed consent for participation in clinical trials (Review) 


\section{B A C K G R O U N D}

Informed consent is a critical stage in the conduct of clinical research (WMA 2000). Several authors have reported that individuals who have given their 'informed consent' do not fully understand their rights as participants, or the methods of their treatment allocation in trials (Hietanen 2000; Harth 1995; Williams 2003). Research also shows that misconceptions about the purpose of a trial and the treatment that will be received are common (Joffe 2001a), and a significant proportion of people do not recall consenting to participation on their own or their child's behalf (Ballard 2004; Chappuy 2006; Schats 2003; Tait 2003). The informed consent process must meet regulatory guidelines for clinical research: nevertheless, poor participant understanding of the research and their participation in it has been demonstrated across clinical areas (Chappuy 2006; Joffe 2001a; Williams 2003). As a result there have been calls for research into innovations to improve the informed consent process for clinical research (Joffe 2001a; Lavori 1999; Tait 2003; Williams 2003).

Different strategies have been tried to improve the informed consent process, such as written information materials and consent forms modified with simpler language, added illustrations, and altered layout to highlight important points (Campbell 2004, Dresden 2001; Kruse 2000). Other studies have included additional detailed verbal and/or written information (Stanley 1998) and computer-based enhancement of information provision (Dunn 2001a) in attempts to improve the process. The use of audio-visual interventions such as informational videos may enhance the informed consent process for clinical research. Audiovisual presentations can ensure the clear delivery of information that is complete, consistent and unbiased, to supplement or reduce staff time spent in seeking informed consent. A recent study of the feasibility of using multimedia technology during the informed consent process for clinical research reported that consumers felt the multimedia tool allowed them to control the pace at which they viewed the information, and that the use of the video 'made information more understandable' (Jimison 1998). Using a visual format to present information on clinical research may complement the informed consent process and audio-visual presentation may be used to provide a dramatic representation of what participation in the study involves. In addition, complex concepts such as randomisation may be explained using visual examples, which may improve potential participants' understanding. The use of an audio-visual format may also enable subgroups of potential research participants, such as those who have difficulty reading complex information, to access and better understand these complex concepts (Campbell 2004). In particular, audio-visual information presented via the Internet, computer or digital video disc (DVD) may promote interactivity, with viewers being able to access specific information that they require, or lay-language definitions of terms, via interactive menus.

Despite the potential benefits of using audio-visual interventions in the informed consent process, there may also be harms associated with their use. In many situations, such as in cancer trials or where parents' consent is sought for a child's participation, informed consent is associated with difficult treatment decisions (Miller 2005; Ruccione 1991; Stryker 2006). Such situations may be inherently distressing, and the information disclosed in the informed consent process may increase people's anxiety or sense of powerlessness (Miller 2005, Ruccione 1991; Simon 2001). People's satisfaction with the informed consent process and with their decision about participating in the research, and their level of decisional regret may also be affected (Franck 2007; Kupst 2003; Pope 2003, Stryker 2006).

With informed consent for trial participation, as for screening and treatment decisions, there is tension between ensuring consumers are informed and ensuring uptake/participation (Edwards 2006; Raffle 2001). In other health arenas, enhancing the structure and content of healthcare information has been shown to result in consumers adopting more conservative screening or treatment choices (O'Connor 2003). The effect of audio-visual interventions for informed consent on outcomes such as rates of trial participation and willingness to participate, is important in this context. For individual consumers, trial participation may be a benefit or a harm, depending on the trial and its particular risks and benefits to them, as well as on their understanding of these underlying risks and benefits. Triallists seeking ethically to provide highquality evidence in order to genuinely benefit society through improved healthcare policy and practice have a legitimate interest in promoting trial participation; while in the context of poorly or unethically-conducted trials, willingness to participate should be considered a harm. In this review we considered it important to capture the audio-visual intervention's effects on people's decision or willingness to participate in the subsequent trial (and the related outcome of their perceptions of the value of the subsequent trial). We emphasise, however, that these outcomes may be either beneficial or harmful to participants and should be considered in the context of the particular trial.

There are other risks associated with the use of audio-visual interventions in this context. Research suggests that social characteristics of the actors in multimedia interventions for informed consent may influence people's attitudes and preferences in relation to medical treatment in important ways, and that this may affect participants' subsequent decision-making (Lenert 2000). Cultural or gender bias towards actors or 'patients' in multimedia presentations may affect the viewers' preferences for health states (Lenert 2000). For example, people rate health states as less preferable when those presenting information are of a different ethnic or cultural group to themselves. They may be less willing to take risks to obtain benefits when the actor in the presentation is female rather 
than male (Lenert 2000). The 'usefulness' of audio-visual interventions may also differ by gender, ethnicity and age: a study of video recorded information for individuals with cancer suggested that younger patients ( $<60$ years of age) and those from ethnic minorities showed a strong preference for video presentations and were more likely to rate the video as 'helpful' or 'very helpful' compared with other participant groups (Thomas 1999).

Informed consent for clinical trials may require a more complex consent process than informed consent for clinical treatment. Terminology used in the consent process for trials may be complex, with concepts such as randomisation, blinding of treatment allocation and equipoise (a state of genuine uncertainty as to the potential benefits and harms of interventions in the trial) needing to be understood. It is ethically imperative that potential trial participants are properly informed and understand that their initial and continuing participation is voluntary (Sugarman 1998). Participants should be told of the aims of the research, the methods that are to be used, the anticipated benefits and potential harms of participation for them as individuals, any anticipated discomfort associated with participation, sources of funding, potential and actual conflicts of interest and the institutional affiliations of the researcher(s) (WMA 2000). In addition, potential participants should be informed of their right to refuse participation or withdraw their consent at any time, without affecting the care they receive. They should also be advised about any costs that they may incur, such as additional travel and parking costs associated with attending hospital more often.

\section{Relationship to other relevant reviews}

This review complements a number of related systematic reviews on informed consent and trial recruitment, some of which focus on improving participant recruitment rates. Mapstone has assessed the effects of interventions to improve recruitment to research studies (Mapstone 2007). Two of the studies included in Mapstone's review investigated changing the informed consent process to improve participation rates, although neither examined providing information by different media. Rendell 2007 investigated the effect of disincentives and incentives on the extent to which clinicians invite eligible patients to participate in trials of healthcare interventions, and identified eleven observational studies. This review identified a range of potential incentives and disincentives, including patient factors such as age, gender, disease type and severity, and ethnicity, but found no consistent effects of these factors on clinicians' willingness to invite people to participate in trials. The authors noted that further research is needed to identify interventions that effectively increase the extent to which clinicians invite people to participate in trials, and that, among other interventions, those to enhance discussions about research concepts (such as randomisation, uncertainty and informed consent) may be useful.
Other relevant systematic and non-systematic reviews have focussed on aspects of the informed consent process for trial participation. Agre, in a non-systematic overview of informed consent research (Agre 2003a) described eight studies investigating the effects of audio-visual (video and computer-based) interventions aiming to improve different aspects of the informed consent process, including participant understanding and informed decision-making. Agre noted that most interventions did not significantly improve knowledge or satisfaction with the decisions made, or influence the decisions made by participants. Computerbased and simplified print interventions were noted as potentially promising, but Agre also found that no single way of presenting informed consent information was clearly superior to other forms across different groups of participants. Flory 2004 systematically reviewed interventions to improve research participants' understanding of informed consent, identifying 12 video and multimedia interventions assessed in nine studies. Only three of these interventions significantly improved participants' understanding. Although two studies showed improved retention of information at follow-up there was little effect of multimedia interventions on individuals' willingness to participate or their satisfaction with the informed consent process. More recently, Cohn 2007 systematically reviewed the literature with a focus on participant comprehension of informed consent in clinical research. This review evaluated the effects of a range of interventions to improve the informed consent process, including simplified written consent forms, use of trained professionals and multimedia approaches. The two studies on multimedia interventions in Cohn 2007 were also included in Flory 2004, with Cohn and colleagues noting that no single approach led to consistent improvements in participants' comprehension associated with the informed consent process.

\section{Why it is important to do this review}

Although several systematic reviews exist in this area, the current review is the most recent and is the only Cochrane systematic review evaluating the effects of a multimedia intervention, specifically audio-visual interventions, on potential clinical trial participants. We used rigorous methods and extensive searching to assemble and evaluate the evidence on the effects of these interventions on potential clinical trial participants.

\section{O B J E C T I VES}

To assess the effects of providing audio-visual information alone, or in conjunction with standard forms of information provision, to potential clinical trial participants who are in the process of seeking informed consent, in terms of their satisfaction, understanding and recall of information about the study, level of anxiety and their decision whether or not to participate. 


\section{METHODS}

\section{Criteria for considering studies for this review}

\section{Types of studies}

Randomised and quasi-randomised controlled trials.

\section{Types of participants}

Individuals or the guardians of individuals who were asked to participate in a clinical trial.

Studies in which the individuals eligible to participate in the trial were children, or were not competent to provide informed consent, and in which parents or surrogates would therefore have provided informed consent on their behalf, were eligible for inclusion.

We excluded studies in which participants were asked to participate in a hypothetical clinical trial: that is, where participants (such as students asked to imagine participating in a trial) were not potential research subjects; or the trial itself was hypothetical (for example, HIV vaccine). Our rationale for this was that outcomes in hypothetical clinical trial participation may not be directly applicable to real-life situations: we sought evidence to inform practice on the informed consent process where potential participation in a trial was a real option for the individuals involved.

\section{Types of interventions}

We included interventions using audio-visual information alone, or in addition to standard forms of information provision (such as written or oral information as usually employed in the particular service setting), compared with standard forms of information provision alone in the process of seeking informed consent from potential participants of clinical trials.

Audio-visual presentation was defined as any pre-recorded audiovisual material presented on the Internet, or by DVD, video or other means.

\section{Types of outcome measures}

The following outcome measures (which included harms of the intervention) were considered to address the needs of both consumers of clinical research and clinical researchers.

\section{Primary outcomes}

1. Participant/guardian satisfaction with information provided about the clinical study.

2. Participant/guardian satisfaction with media used to convey the information about the clinical study (including the ability for interaction).

3. Participant/guardian knowledge and understanding of the clinical study including: the potential harms; the potential benefits; treatment(s) involved (if relevant); the outcomes assessed and the data collected; the need for and duration of follow up; the concept of equipoise (a state of genuine uncertainty as to the potential benefits and harms of interventions in a clinical study); the concept of randomisation (if relevant); understanding of the use of a placebo (if relevant); their right to refuse participation and their right to withdraw from the study at any time.

4. Retention of participant's/guardian's knowledge and understanding of clinical study (including: the potential harms; the potential benefits; treatment(s) involved (if relevant); the outcomes assessed and the data collected; the need for and duration of follow up; the concept of equipoise; the concept of randomisation (if relevant); understanding of the use of a placebo (if relevant); their right to refuse participation and their right to withdraw from the study at any time); measured two or more weeks after viewing the video or receiving the standard information.

5. Anxiety of participant/guardian associated with the informed consent process (variously defined and measured by the authors).

6. Participant/guardian satisfaction with the decision-making process; whether they felt they made the decision themselves; whether they were satisfied with their choice to participate or decline participation in the study; whether, given their time again, they would choose to participate or decline participation in the study (within two years of being asked to participate in the clinical study); whether they regretted their decision to participate or decline participation in the study (within two years of being asked to participate in the clinical study); whether they would advise others to participate or decline participation the study.

7. Rate of participation or willingness to participate in the clinical study.

\section{Secondary outcomes}

1. Level of adherence to the study protocol for participants who entered the study.

2. Rate of withdrawal from the study following consent for study participation.

\section{Search methods for identification of studies}

We conducted searches in April 2004 and updated the searches in June 2006. There were no language or date restrictions. We provide full search strategies for each database in Appendices 1 to 9 .

\section{Searches in 2004}

In April 2004 we searched the following databases: 
- Cochrane Consumers and Communication Review Group Specialised Register (searched 28 April 2004);

- Cochrane Central Register of Controlled Trials

(CENTRAL), The Cochrane Library, issue 2, 2004;

- MEDLINE (Ovid) (1966 to April week 1 2004);

- EMBASE (Ovid) (1988 to 2004 week 15);

- PsycINFO (Ovid) (1974 to April week 2 2004);

- CINAHL (Ovid) (1982 to week 2 2004);

- Current Contents (Ovid) (1993 to 2004 week 18); and

- ERIC (CSA Illumina) (searched 28 April 2004).

We had planned to search a number of additional databases as specified in the review protocol, but decided that complete coverage of the area was already ensured by the above databases. The databases specified in the protocol but not searched for this review were: PsycLIT and Psychological abstracts (covered by PsycINFO); Healthstar (covered by MEDLINE); CONSUMER; Consumer Sciences Index; Consumer reports on health; Current index to journals in education (CIJE); Resources in education (RIE); Institute for Scientific Information; ISI Web of Science (searched only as secondary citation index).

\section{Searches in 2006}

We re-ran searches on 20 June 2006 using the strategies contained in Appendices 1 to 3 and 5 to 9. We checked the 2004 strategies and amended the PsycINFO strategy to reflect changes to subject headings. (Both versions of the PsycINFO strategy (2004 and 2006) are presented, in Appendices 4 and 5 respectively). We restricted the updated searches to the years 2004 to 2006, and searched the following databases:

- Cochrane Consumers and Communication Review Group Specialised Register (searched 20 June 2006);

- Cochrane Central Register of Controlled Trials

(CENTRAL), The Cochrane Library, issue 2, 2006;

- MEDLINE (Ovid) (1966 to June week 1 2006);

- EMBASE (Ovid) (1988 to 2006 week 24);

- PsycINFO (Ovid) (1967 to June week 2 2006);

- CINAHL (Ovid) (1982 to June week 3 2006);

- Current Contents (Ovid) (1993 week 27 to 2006 week 26); and

- ERIC (CSA Illumina) (searched 20 June 2006).

We searched reference lists of included studies and relevant review articles, and contacted trial authors and other experts (see Appendix 11).

\section{Data collection and analysis}

\section{Selection of studies}

Two review authors (KM and RR) independently screened the titles/abstracts of studies identified by the searches against the inclusion criteria. We retrieved in full text all studies identified from their titles as being potentially relevant, and the same two authors assessed them for eligibility. Discrepancies were resolved by discussion with a third team member (CC/MP). Studies excluded at this stage are listed in the table Characteristics of excluded studies, with reasons given.

\section{Data extraction and management}

For included studies, two authors (RR and KM/MP) independently extracted detailed descriptive data, with discrepancies resolved by discussion and consensus. Data collected from each study was based on the Cochrane Consumers and Communication Review Group Data Extraction Template (CCRG 2004), and included the following: authors and year of publication, setting, country, time span of the trial, pretrial calculation of sample size, participant characteristics and numbers (recruited, randomised and analysed) and details of the analysis performed (see table Characteristics of included studies).

We extracted detailed information about the interventions, including: the length, quality and language used; content; medium (eg. video, DVD, videodisc or Internet); setting where the audiovisual information was viewed; the number of times it was viewed; whether it was viewed individually or as part of a group; whether the audio-visual information was interactive or non-interactive; and whether the audio-visual information was viewed alone, or was provided in conjunction with other forms of information provision (co-interventions). We also extracted the standard information (usual care) provided in each of the trials (see Additional Table $1)$.

We extracted detailed information on the outcomes assessed by each study, including each outcome measured; the method and timing of measurement; and the validity and reliability of the measure used (see Additional Table 2).

\section{Assessment of methodological quality of included studies}

We assessed the methodological quality of included studies based on the following items (see also Additional Table 3):

- method and adequacy of randomisation (Higgins 2005);

- method and adequacy of allocation concealment (Higgins 2005);

- blinding of participants, intervention providers

(investigators, study nurses) and outcome assessors;

- completeness of participant follow up;

- baseline comparability of intervention and comparison (control) groups;

- validity of outcome measures used;

- reliability of outcome measures used;

- intention-to-treat (ITT) analysis performed. 
Two review authors independently assessed each item, rating it as 'yes' (clearly done), 'no' (clearly not done) or 'unclear' (not enough information provided to clearly decide whether it was or was not done) (Ryan 2007).

The protocol for this review stated that we would assess information on completeness of follow-up in each study, according to guidance contained in Clarke 2001. After discussion, and owing both to the small number of included studies and the apparent lack of clear consensus on what comprises 'adequate' follow-up, we decided to report instead the number and percentage of participants followed up.

Assessments of the validity of outcome measures was based on: whether any attempt to validate the outcome measure was reported (in the trial report or by trial authors upon contact) and the results of the validation; or whether the outcome measure was an established measure for the outcome under consideration, with appropriate references cited to confirm that validity had been established in the trial context. Outcome measures were rated as validated ('yes') if one or both criteria were met; unvalidated if neither were clearly met ('no'); or 'unclear' if there was not enough information to decide whether either criteria had been met.

We took a similar approach in assessing reliability of outcomes. Assessment was based on any reported attempt to assess the reliability of the outcome measure; and the level of reliability reported for the outcome. Outcome measures were assessed as: 'reliable', if high levels were reported (eg. alpha above 0.80) ('yes'); 'not reliable', if low levels were reported (eg. alpha below 0.80 ) or there was no attempt to assess reliability ('no'); and 'unclear', if there was not enough information to decide whether either criteria had been met.

\section{Data synthesis (meta-analysis)}

We had planned to meta-analyse data from included studies, but because of the small number of studies and the diversity of outcome measures and participant groups we decided to present the results narratively. Details of the planned meta-analysis methods (including sensitivity and subgroup analyses) are retained in Appendix 10 for use in future updates of this review.

\section{Author and expert contact}

Between December 2004 and December 2006 we contacted authors of included and some possibly-eligible studies, and experts in informed consent, in order to: gather additional information on the included studies; confirm inclusion/exclusion of eligible studies; and identify other potentially-relevant studies both published and in progress. We present details of author contact in Appendix 11. Authors of Agre 2003b; Benson 1988; and Weston 1997 provided substantial additional information. We were unable to contact authors of Norris 1990.

\section{Consumer participation}

For each included study, we collected information (from the trial report and from author contact where possible) about the involvement of consumers in the development and evaluation of the interventions. We also sought literature on consumers' views of informed consent for clinical studies, and their needs from the informed consent process. This literature helped to inform the review, particularly the Discussion, and the recommendations for further research.

Consumer advocates assessed both the protocol and the review, to ensure that consumers' needs and views were represented appropriately.

\section{RES U L T S}

\section{Description of studies}

See: Characteristics of included studies; Characteristics of excluded studies.

\section{Results of the searches, and excluded studies}

\section{4 searches}

Electronic searches in 2004 identified 2743 titles, with 2507 remaining after duplicates were removed using Endnote X software. A further six papers were identified through secondary sources (the reference lists of included studies or of reviews).

We screened 2513 citations, and of these obtained 51 potentiallyrelevant papers in full text for further examination. Of these, three studies (Dunbar 1989; Weston 1997; Wirshing 2005) were each reported in two papers. Of the 48 studies remaining, we excluded 46 studies for various reasons (see table Characteristics of excluded studies). We considered one of these studies (Fureman 1997) at length before excluding it on the basis that it examined informed consent for a hypothetical (rather than actual) HIV vaccine trial. The remaining two studies (reported in three papers) were included in the review (Norris 1990; Weston 1997).

Agre's overview of informed consent research (Agre 2003a), located through the electronic searches, led us to five other potentiallyeligible studies (Agre 2002; Agre 2003b; Mintz et al; Kass; Sachs 2003). Two (Agre 2002; Sachs 2003) were overviews of informed consent research and hence excluded. We included Agre 2003b. Mintz et al was a duplicate report of Wirshing 2005. Kass may be eligible for inclusion but complete data are not yet available, so we will assess the study in a subsequent update of this review. We identified, but then excluded, three additional studies identified through author contact (Agre 1994; Dunn 2001a; Hougham 
2003). Two further potentially-relevant (un-cited) studies were described in the overview by Hougham 2003 but cannot be assessed for inclusion in the review without further information.

\section{6 searches}

The searches updated in 2006 generated 394 citations, of which 290 remained for assessment after duplicates were removed using EndNote X software. We identified three papers as potentially relevant and retrieved them in full text (Campbell 2004; Flory 2004; Wirshing 2005) but ultimately excluded them (see table Characteristics of excluded studies).

From the reference list of Flory 2004 we identified two studies which were retrieved in full text: Tindall 1994, which was excluded, and Benson 1988 which was included in the review. We identified two potentially-relevant studies incidentally as the review was being finalised (Joseph 2006; Moseley 2006); both were excluded.

\section{Included studies}

We included four studies (Agre 2003b; Benson 1988; Norris 1990; Weston 1997) reported in five papers. Detailed information about these studies is presented in the table Characteristics of included studies, and in Additional Table 1, 'Details of Interventions', Table 3, 'Quality of Included Studies', and Table 2, 'Details of Outcome Measures'.

\section{Design}

Three of the four studies were randomised controlled trials (Agre 2003b; Norris 1990; Weston 1997). The fourth (Benson 1988) was a quasi-randomised controlled trial which sequentially allocated participants to study groups.

Two of the four included studies compared the audio-visual intervention plus usual care with usual care alone (Norris 1990; Weston 1997). In both cases, usual care included some form of written information (pamphlet or consent form), as well as some form of verbal information (discussion with the study nurse (Norris 1990); availability of the study nurse to answer participants' questions (Weston 1997)).

Of the other two studies, Agre 2003b examined the effects of four different formats for presenting informed consent information (booklet, video, computer-aided instruction and standard written consent form). For this review, we only considered information related to the video and computer-aided instruction interventions, compared with standard consent information (that is, we did not consider the booklet intervention: this represented a non-multimedia, non-standard informed consent intervention and so was outside the scope of the review).

Benson 1988 also examined the effects of four different formats for presenting consent information: two different video and instructor-provided information arms, a 'neutral educator' arm and a routine informed consent procedure. For this review, we did not include data from the neutral educator intervention as this represented a non-multimedia, non-standard intervention that was outside the scope of the review.

\section{Sample sizes}

Studies ranged in size from 88 to 441 participants; in total 819 people participated in the four studies. Of these, we included data from potential participants in research trials (not surrogates); and analysed data from control and audio-visual intervention arms only. In total, we included data from 511 participants in this review.

\section{Geographic location and setting}

At least two of the included studies were conducted in the USA (Agre 2003b; Benson 1988). The location of Norris 1990 was not specified but appears likely to have been Tennessee, USA. Weston 1997 took place in Canada.

Three of the studies were based in hospitals/medical centres. In a fourth study (Norris 1990) the setting was unspecified.

\section{Participants}

Participants differed across the four included studies, reflecting the diverse trials for which informed consent was being sought: specifically, a series of 18 oncology trial protocols (Agre 2003b); a group of psychiatric research studies (Benson 1988); a clinical trial of duodenal ulcer medication (Norris 1990); and a clinical trial of the management of pre-labour rupture of membranes (PROM) at term (Weston 1997).

Agre 2003b recruited cancer patients considering participation in one of 18 oncology trials, their accompanying family members, and non-patient surrogate subjects (individuals waiting for patients in the Day Hospital waiting room). For this review, we have only included data on patients. Data for non-patient surrogates was excluded on the basis that it represented hypothetical clinical trial participation. Data for family members/ friends accompanying patients was also excluded, as their role was not expressly to give informed consent on the patient's behalf. The patients included in this study were predominantly male (74\%) with an average age of 59.5 years, and a generally high level of education. No information on participants' ethnicity was reported for the patient subgroup, although of the entire study sample only $12 \%$ were of ethnic minority status, predominantly African American or Latin American.

Benson 1988 recruited psychiatric patients as they were identified as prospective research participants for four existing research studies on schizophrenia, depression, social skills and borderline personality disorder. There were three diagnoses among those recruited to the informed consent study: $50 \%$ had a diagnosis of schizophrenia, $27 \%$ depression and $23 \%$ borderline personality 
disorder. Most study participants were male (70.5\%) and white (70\%); their average age was 41 years and they had, on average, 12 years of education.

Norris 1990 recruited individuals to participate in a clinical trial of duodenal ulcer medication. The source of these participants was unclear and Norris gave no information on their demographic or other characteristics. The study reports participant numbers inconsistently, stating initially that 278 participants signed the consent form and were randomised, but later that 200 participants were randomised to two groups. As we could not contact the authors we have adopted a conservative approach and assumed that 200 participants were assigned to two groups and studied. Weston 1997 recruited pregnant women from physicians' offices and clinics to a trial of informed consent to a trial evaluating the management of Term Prelabour Rupture of the Membranes ('Term PROM'). These women were aged between 18 and 39 years and were between 19 and 33 weeks' gestation. At this stage it could not be known whether their membranes would rupture before labour, making them eligible for the Term PROM trial itself. They were recruited specifically at an earlier gestational age (rather than at full term) so that the informed consent study did not interfere with recruitment to the actual Term PROM trial: the participants of this informed consent trial therefore represent future potential trial participants (that is, women who may in the future consider actual trial participation should they become eligible). Educational level was generally high, with over $90 \%$ of participants having at least a college education. No information on their ethnicity was reported.

\section{Interventions}

Despite the relatively narrow definition of 'audio-visual information' interventions adopted for this review, there was considerable variation in the content, design and delivery of interventions examined by the included studies. We present detailed information on interventions (as well as co-interventions and usual care) in the table Characteristics of included studies and Additional Table 1. Two of the four included studies assessed the effects of an audio-visual intervention(s) in addition to usual forms of information provision (Norris 1990; Weston 1997). In Agre 2003b, participants were allocated to receive one of two audio-visual interventions (video or computer-assisted instruction), or the standard written consent form. In Benson 1988, participants were also allocated to receive one of two audio-visual interventions (in this case two forms (standard and improved) of an instructional video), or to the standard informed consent disclosure.

Agre 2003b examined the effects of video and computer-aided instruction interventions in comparison with the standard written consent form. The standard consent form for each oncology protocol served as the basis for development of scripts for the professionally-produced video and computer-assisted instructional program. All interventions included identical sections based on the standard informed consent procedure for clinical trials at the insti- tution. This included sections outlining information on: the purpose of the research procedures; the side effects, risks and benefits of treatment; alternatives; financial costs; compensation in the case of research-related injury; confidentiality; and the right to refuse or withdraw. The actual content of the interventions was tailored to the research protocol and was based on the oncology trial for which consent was being sought. The video/computer interventions were of varying length (range: 10.24 to 21.39 minutes) depending on the oncology protocol. Video/computer interventions were viewed or used once only, in private consultation rooms, by patients, their families and non-patient surrogates recruited to the trial. Usual care involved review of the written consent document. Benson 1988 assessed the effects of two different videos, termed 'instructional' (standard) and 'improved', compared with routine information presented by psychiatric researchers. Video interventions were developed by the research team, and not professionally produced. In the standard video the principal investigator or other project staff member described the study as s/he chose to. Feedback from the research team on areas of the disclosure that could be improved, or required greater emphasis, was incorporated into production of the second 'improved' video format. No information was available on the video length nor on the setting in which videos were viewed. Usual care was routine information provided as per the psychiatric researcher's standard disclosure to trial participants.

Norris 1990 used a video of unspecified length and duration, which included information on trial medication compliance issues, follow-up procedures, potential adverse effects and study consent forms. No further information about the delivery or setting of the video interventions was available. Usual care involved potential participants studying the consent form, followed by discussion with the study nurse.

Weston 1997 used a professionally-produced video which included interviews with patients, their families, nurses and the study's principal investigator and staff. Ten minutes long, the video described the medical condition (pre-labour rupture of membranes at term) and details of the trial, including the intervention, its risks and benefits, aspects of the trial protocol and an interview with an actual trial participant). The video was viewed by individual women at the doctor's office or in their homes. Usual care included written information with questions answered by the study nurse.

\section{Outcomes and the measures used}

Three of the four included studies used questionnaires completed by participants to assess outcomes. The fourth, Benson 1988, used participant interviews and observation of disclosure sessions to evaluate outcomes. We present details of all outcomes in the table Characteristics of included studies and Additional Table 2.

The included studies assessed only a limited range of outcomes. None reported using formalised, validated measures of informed consent or decisional capacity, such as the MacArthur Compe- 
tence Assessment Tool for Clinical Research (Dunn 2006) or the Quality of Informed Consent questionnaire (Joffe 2001b). As all interventions aimed to increase participants' knowledge and/or understanding of the trial for which their informed consent was sought, each of the included studies measured this outcome, but using different measures. Agre $2003 \mathrm{~b}$ used self-completed multiple choice knowledge quizzes containing 12 to 15 items, which were created for each of the oncology protocols to assess participants' knowledge and understanding of the trial, based on the proportion of correct responses. Benson 1988 used interviews to assess participants' understanding of research based on responses to 15 items. Norris 1990 used a 10-item patient consent quiz to assess knowledge of the information contained in the patient information sheet. Weston 1997 assessed two sets of knowledge using a single questionnaire. Five questionnaire items assessed knowledge of the study protocol, with a further six items assessing knowledge of the health condition (Term PROM).

Agre 2003b and Norris 1990 did not report data on other outcomes. Benson 1988 assessed the quality of the research information disclosed to participants; completeness of the information items communicated was rated on a four-point scale by an observer of the disclosure session, using the study's written consent form as the standard reference point. Weston 1997 evaluated the willingness of participants to participate in the Term PROM trial, and the perceived importance (worth) of the trial. Each of these outcomes in Weston 1997 was assessed via a single item on the self-completed questionnaire.

The timing of outcome assessment varied across included studies. In Agre 2003b and Norris 1990 knowledge was assessed at a single time point, immediately after the informed consent intervention was delivered. Benson 1988 and Weston 1997 also assessed outcomes immediately after the intervention, but repeated this measure at later times: approximately two weeks later for Benson 1988 (although data collected at this later time point was not reported), and between two and four weeks post-intervention for Weston 1997.

Several outcomes specified in the protocol of this review were not assessed by any of the included studies. These included the following primary outcomes:

- participant/guardian satisfaction with information provided about the clinical study;

- participant/guardian satisfaction with media used to convey the information about the clinical study;

- anxiety of participant/guardian associated with the informed consent process;

- participant/guardian satisfaction with the decision-making process.

Neither of the secondary outcomes specified in the protocol were assessed by the included studies, namely:

- the level of adherence to the study protocol for participants who entered the study;

- the rate of withdrawal from the study following consent for study participation.

\section{Consumer involvement}

Agre 2003b and Benson 1988 reported that consumers were not involved in the development of the intervention. Norris $1990 \mathrm{did}$ not report sufficient information to assess whether consumers were involved. Weston 1997 included consumers in the production of the audio-visual (video) intervention. The video included dialogue with an actual trial participant discussing her reasons for participating in the trial and the contribution that she felt she had made to future women and to medical science.

\section{Funding sources}

Two of the studies did not report any sources of funding. Benson 1988 reported funding from the Foundations' Fund for Research in Psychiatry, and Weston 1997 reported funding from the Medical Research Council of Canada. There is no indication that any studies received industry funding.

\section{Risk of bias in included studies}

We assessed the methodological quality of included studies as specified in 'Methods of the Review'. We present details of the quality assessment in Additional Table 3.

Norris 1990 provided insufficient detail about study design and execution, and hence is rated as 'unclear' on most methodological quality items; we note that this may be a reflection of inadequate reporting rather than of poor conduct of the trial itself. The three remaining studies attempted to minimise at least some potential sources of bias, and reported their methods in some detail. Agre 2003b and Benson 1988 achieved adequate quality in several areas, including follow-up of participants, use of intention-to-treat (ITT) analysis and baseline comparability of groups. Weston 1997 was of comparatively higher quality, with adequate and well-described methods of randomisation and allocation concealment, and with adequate participant follow-up and use of ITT analysis. None of the included studies adequately achieved blinding (of participants, intervention providers or outcome assessors) at the point of allocation to groups, intervention delivery or outcome assessment. Nor were outcome measures formally validated (see Additional Table 2). The variable quality of included studies overall suggests that their results, both individually and collectively, should be interpreted with caution. Components of the quality assessment are summarised below.

\section{Method and adequacy of randomisation and allocation concealment}

Only Weston 1997 used a truly random number sequence and concealed allocation adequately. Agre 2003b reported adequate methods of randomisation but did not conceal allocation. Benson 
1988 sequentially allocated people to groups without concealing allocation. Norris 1990 did not report methods of randomisation or allocation concealment.

\section{Blinding}

None of the included studies took adequate steps to achieve blinding of participants, intervention providers or outcome assessors. None of the studies reported adequate measures to ensure blinding either at the point of participant allocation or at the point of intervention delivery. Blinding at the point of allocation may be possible, but the nature of audio-visual interventions may make blinding of participants (recipients) and providers difficult or impossible at the time of intervention delivery. This represents a possible source of bias in the included studies if either participants or providers have a preference for either arm of the trial.

Measures to ensure blinding of outcome assessment were likewise inadequate. Blinding of outcome assessors may not be possible in situations where self-reported outcome measures are used and participants are not blind to their allocation, as appeared to be the case for three of the four included studies (Agre 2003b; Norris 1990; Weston 1997). Benson 1988 assessed outcomes by observing the informed consent disclosure sessions and participant interview and for these outcomes it may have been possible to blind assessors to the audio-visual intervention arms (although not to the usual care arm). Overall, the lack of blinding in these included studies represents a limitation of all studies that may predispose them to bias.

\section{Completeness of follow-up}

Follow-up and reporting of outcomes for recruited participants was high across all included studies. Agre $2003 \mathrm{~b}$ reported outcomes for all 155 (100\%) patient participants allocated to the control group and either of the intervention arms (video or computeraided instruction) considered by this review. Likewise Benson 1988 reported outcomes on all 66 participants allocated to the control or two intervention arms (standard video and improved video interventions) considered by this review. In Norris 1990 outcome data was reported as percentages, implying complete followup of the 200 enrolled participants, although this was not explicitly stated. Weston 1997 reported that all 90 (100\%) participants completed the baseline questionnaire and 85/90 (94\%) completed the questionnaire at two to four weeks post-intervention.

\section{Baseline comparability of groups}

Three of the four studies reported that groups to be studied were comparable on key characteristics at baseline and reported data on these key characteristics (Agre 2003b; Benson 1988; Weston 1997). Norris 1990 did not report participant characteristics and was rated as unclear on this item.

\section{Method of analysis: intention-to-treat (ITT)}

Three of the four included studies analysed results on an ITT basis (Agre 2003b; Benson 1988; Weston 1997). Norris $1990 \mathrm{did}$ not perform any statistical analysis, reporting percentage data for correct responses only.

\section{Validity of outcome measures}

We provide details of each outcome measured by included studies in Additional Table 2. None of the studies formally validated the outcome measures used. Face validity for knowledge/ understanding was assessed informally by the principal investigator in Agre 2003b (confirmed by author contact). The validity of outcome measures used by Benson 1988 and Norris 1990 was unclear, and author contact confirmed that outcome measures used by Weston 1997 were not formally validated.

\section{Additional study design issues}

Three of the four included studies performed pre-trial power calculations (Agre 2003b; Benson 1988; Weston 1997). For Benson 1988, delays in recruitment reduced participant numbers below prespecified levels in two of the four component psychiatric trials. Reliability of outcome measures was assessed formally in only one study: Benson 1988 reported high reliability for both outcomes assessed (in each case alpha $>0.80$ ). Contact with the authors of Agre 2003b confirmed that reliability of the outcome measure had not been formally assessed, but that since outcomes were assessed verbatim it was unlikely to vary according to the assessor's interpretation of participant responses. Reliability of outcome measures was unclear in the study by Norris 1990 , and was not assessed in Weston 1997.

\section{Effects of interventions}

Despite the small number of studies included and the well-defined nature of the intervention, there was considerable variation between studies in terms of the purpose of the trial for which informed consent was sought, and hence in the development and content of the interventions. The populations studied and the outcome measures used also varied. As a result we did not consider it valid or useful to pool the results of these studies statistically. All four included studies assessed knowledge and/or understanding of the subsequent trial for which informed consent was sought. The remaining outcomes were assessed by single studies. We describe below the results, organised by the outcomes for which data were available.

\section{Knowledge and/or understanding of the trial to which informed consent was sought}


Overall, the four studies reported inconsistent effects of audiovisual interventions on knowledge/ understanding levels, compared with usual care: two reported no significant differences in knowledge/ understanding (Agre 2003b; Benson 1988), one an increase in knowledge (Norris 1990), and one reported no difference immediately following the intervention, but better retention of knowledge in the intervention group compared to the control group two to four weeks later (Weston 1997).

Agre 2003b assessed participant knowledge following a video presentation, computer-aided instruction or standard written consent information. Knowledge was assessed as the proportion of correct responses on 12- to 15-item multiple choice knowledge quizzes, each specific to the oncology protocol in which the participant was considering enrolling. Knowledge was assessed immediately following the consent intervention. This study reported no overall differences in mean knowledge scores across study groups. The mean knowledge score for participants in the standard consent (control) group was $68.23 \%$ (standard deviation (SD) $19.24 \%$ ); this was lower than the video intervention group (71.00\% (SD 24.70\%)) and comparable to the computer-aided instruction group $(68.01 \%$ (SD 25.58\%)).

Norris 1990 assessed knowledge following either a video presentation or discussion with the study nurse. Knowledge was assessed at an unspecified time, by reporting the number of items correct on a 10-item consent quiz. Eighty-two percent of participants in the video intervention group scored $10 / 10$ on the quiz, compared with no participants in the control group. The remaining $18 \%$ of participants in the video intervention group scored 8 to $9 / 10$ on the quiz, compared with $30 \%$ of the control group participants achieving this score. Of the remaining 70 participants in the control group, 35 (35\%) scored between 4 and $7 / 10$, and 35 (35\%) scored between 0 and $3 / 10$ on the quiz. This study did not report any statistical comparisons between groups.

Weston 1997 assessed women's knowledge following a video presentation or standard written information and discussion with the study nurse. Knowledge was assessed immediately after the intervention and at two to four weeks post-intervention. This study used an 11-item questionnaire to assess content knowledge; 5 items assessed knowledge of the study protocol, and the remaining 6 items assessed knowledge of the health condition involved in the subsequent study. For overall content knowledge, assessed as 9 or more of 11 content questions correct, there were no significant differences between the two groups (intervention group 40/ 42 (95\%) versus usual care group 42/48 (88\%), when assessed immediately after the intervention. When assessed two to four weeks later, women in the video group were more likely to answer nine or more of the knowledge questions correctly $(36 / 41,88 \%)$, compared with women receiving usual care $(28 / 44,64 \%$; $\mathrm{P}=0.01)$.

This study also reported numbers of correct responses to each of the 11 content questions individually for the video and usual care groups, at baseline and at follow-up. Authors did not statistically compare these numbers but did note that no specific content ques- tion or subject area appeared more difficult than any other for the women involved in the study. Knowledge of the health condition (Term PROM) was assessed with six of the 11 content questions. The pattern of results was similar to that seen for overall knowledge levels. At baseline, women in the intervention and usual care groups reported similar levels of knowledge of Term PROM (intervention group: 235/252 (93\%) questions answered correctly, versus usual care group: 261/288 (91\%) answered correctly). Two to four weeks later, women in the intervention group reported slightly higher levels of knowledge of the health condition than those receiving usual care (intervention group: 209/246 (85\%) questions answered correctly, versus usual care group: 207/264 (78\%) answered correctly).

Knowledge of the study protocol was assessed by the five remaining content questions in Weston 1997. Again, the pattern of results for study protocol knowledge was similar to that seen for knowledge overall. At baseline, women in the intervention and usual care groups correctly answered similar numbers of questions on the study protocol (intervention group: 202/210 (96\%) questions answered correctly versus usual care group 220/240 (92\%) questions answered correctly). At follow up, women in the intervention group reported slightly higher knowledge levels of the study protocol than those receiving usual care (intervention group 192/205 (94\%) questions answered correctly, versus control group: 191/ 220 (87\%) questions correct).

Benson 1988 assessed understanding following presentation of one of two videos or standard informed consent disclosure by researchers. Participants' understanding of research was measured at two time points: immediately following the disclosure session and approximately two weeks after the disclosure session (note that only data for the first time point was reported). Understanding was assessed on participants' responses to 15 items, each rated on a 3 -point scale $(0=$ poor to 2 = fair $)$. The overall composite understanding score was calculated from responses to individual items and calculated to give an overall score ranging from 0 to 30 . Benson only reported data collected immediately after the disclosure session. This study reported no significant differences in mean composite understanding scores across study groups (unassisted disclosure (control) group 15.41 (51.4\%; SD 6.43); unassisted video disclosure (standard video) group 16.05 (53.5\%; SD 6.4); assisted video disclosure (improved video) group 17.27 (57.6\%; SD 6.24). Nor were there significant differences in levels of understanding assessed for each of the individual 15 items across the three study groups.

It is also important to consider the size of the effects of audio-visual interventions on knowledge and/or understanding, compared with routine forms of information provision. Both Norris 1990 and Weston 1997 reported high levels of knowledge, reflected by numbers of correct responses, following the interventions. In both cases, the percentage of participants answering correctly (as 10/ 10 correct answers, or 9 or more correct answers out of 11 respectively) exceeded $80 \%$. In comparison, Agre 2003b reported 
correct responses of approximately $70 \%$ across the control and intervention groups. Similarly, the proportion of correct responses in Benson 1988 was consistently $<60 \%$ across study groups. The findings of Agre 2003b and Benson 1988 are important: they suggest that a large amount of the relevant information may not be understood by potential trial participants, as suggested by previous research. This has implications for the notion of informed consent, discussed later in this review.

\section{Willingness of participants to participate in a future trial}

Weston 1997 assessed participants' willingness to take part in the Term PROM trial. More women in the video group were willing to participate in the trial $(26 / 42,62 \%)$, compared with the usual care group $(17 / 48,35 \%)$ immediately following the intervention $(P=0.01)$. However at two to four weeks post-intervention there was no longer a significant difference between the video and usual care groups (intervention 23/41 (56\%) versus usual care 17/44 (39\%); $\mathrm{P}=0.11)$.

\section{Perceived importance (worth) of the trial}

Weston 1997 also reported the perceived importance (worth) of the subsequent trial as an aspect of 'willingness to participate'. Immediately after the intervention, more women in the intervention group rated the trial as 'very important' $(30 / 42,71 \%)$, compared with the usual care group (25/48, 52\%), but this was not statistically significant $(P=0.06)$. A similar but smaller trend was also observed at two to four weeks post-intervention.

\section{Quality of research information}

Benson 1988 assessed the effects of two video interventions, each compared with standard disclosure methods, on the quality of the research information communicated to potential trial participants during the informed consent disclosure session. Completeness of the communicated information was rated by an independent observer, using a 4-point scale $(0=$ no disclosure of information to $3=$ disclosure of information superior to the written consent form) to evaluate different aspects of the session. Mean global information quality assessments were calculated (range: 0 to 30). The overall mean information quality score for the unassisted disclosure (control) group was 14.95; the score for the unassisted video disclosure (standard video) group was not significantly different, at 18.34 . In comparison, the mean information score for the assisted video disclosure (improved video) intervention was significantly higher (mean 22.80, $\mathrm{P}<0.05$ ) than that in the control group.

Benson also reported analysis of the subcategories of information provided. Compared with the control group, a significant increase in the quality of information communicated to potential trial participants was found for only one of the six subcategories in the unassisted video disclosure (standard video) intervention arm. In contrast, five of the six subcategories showed significant increases in the quality of communicated information when the assisted disclosure (improved video) intervention was used.

As this review only included four studies, we could not conduct the planned subgroup analyses to examine the effects of timing of the audio-visual intervention and outcome assessment, or to assess differences in the intervention recipient (guardians of individuals versus individuals themselves). However, as this is likely to represent a growing research area, more trials may become available to make formal meta-analysis and secondary subgroup and sensitivity analyses possible in future updates of this review.

\section{DISCUSSION}

Despite the importance of informed consent for clinical trial participation, and previous work suggesting that audio-visual interventions may be an effective way of delivering important information to promote consent by trial participants that is informed, little research rigorously evaluates the effects of these interventions. Extensive searching identified only four trials for inclusion in this review. Each of these trials had limitations in their design, conduct or reporting. None of the included studies met all of the methodological quality criteria prespecified for this review: included studies may therefore be predisposed to bias and this may limit confidence in their results, taken either individually or collectively. Both the number of trials, and the total number of participants included in the review, were relatively small; outcomes other than knowledge/ understanding were only reported in single studies. Despite this, some interesting findings have emerged from the included studies.

\section{Summary of the main results}

The effects of audio-visual interventions in the four included studies were variable in size and mixed in direction. As a result, it is difficult to draw firm conclusions about the effects of audio-visual interventions on the limited range of outcomes assessed.

Audio-visual interventions did not consistently significantly increase participants' levels of knowledge/understanding. Two studies reported no significant differences in knowledge/ understanding following audio-visual interventions (Agre 2003b; Benson 1988). One study reported an increase in knowledge, although this was not tested statistically (Norris 1990), and one reported no difference immediately following the intervention, but significantly better retention of knowledge in the intervention group compared to the control group two to four weeks later (Weston 1997).

Audio-visual interventions may briefly increase people's willingness to participate in trials (Weston 1997), although this was reported by a single study and the effect was not sustained at follow- 
up two to four weeks later. In any case, enhanced willingness to participate in trials may be a beneficial or harmful outcome for the potential participant, depending on the balance of possible benefits versus harms for them of participating in a clinical trial, and on their underlying understanding of these potential benefits and harms associated with participation (Robinson 2005; Sugarman 1998). Perceived worth of the trial did not appear to be influenced by an audio-visual intervention (Weston 1997). The quality of information disclosed may be enhanced by an 'improved' video intervention, but it did not significantly improve following a 'standard' video intervention, compared with standard information disclosure (Benson 1988). The heterogeneity in the results may reflect the differences in intervention design, content and delivery, the populations studied and the varied methods of outcome assessment.

\section{Overall completeness and applicability of the evidence}

Considerable uncertainty remains about the effects of audio-visual interventions, compared with standard forms of information provision, for use in the process of obtaining informed consent for clinical trials. This uncertainty encompasses both the fairly narrow range of outcomes assessed by the studies included in this review, as well as the large number of outcomes that were not examined by any of the included studies. These latter outcomes include both primary and secondary outcomes, such as satisfaction with the information provided, with the media used to convey information and with the decision-making process; the level of adherence to the study protocol and withdrawal rates from the study following consent for participation in the trial. Additionally, none of the included studies explicitly assessed potential harms of audiovisual interventions, such as anxiety associated with the informed consent process for clinical trial participation.

One study included women who were potential future trial participants (Weston 1997), and this may also add to the heterogeneity in the results. Participants in included studies were generally well educated, and participation was sometimes restricted to those who could read and write English. No studies assessed the effects of audio-visual interventions on children and/or their guardians, or on other vulnerable groups such as the unemployed, refugees, or people with low education levels, intellectual disability or learning disorders, although psychiatric patients with a range of diagnoses were included in Benson 1988. It is unclear whether the results of this review would be relevant to people who are seriously ill, such as those with acute and life-threatening conditions. There may be creative ways of using audio-visual interventions in such situations, for example, to inform family members while an emergency is in progress. People who are seriously ill may also be more inclined to believe that a trial is designed to benefit them directly: a staged approach to the informed consent process, such as an oral explanation of the research followed up with written consent materials, may be useful in these instances (Wilets 2005).

It appears that none of studies assessed the effects of the intervention in settings outside North America. Since the determinants of informed consent are complex and are not readily generalisable from high income to low-and-middle income countries (Krosin 2006; Verástegui 2006), the effects of audio-visual interventions in other settings are not clear.

We conclude that the included studies provide incomplete evidence for the effects of audio-visual interventions during the consent process for clinical trial participation, and that the applicability of the available evidence is limited. Further well designed and clearly reported trials of this intervention are needed (Brown 2006).

\section{Consumer involvement}

Weston 1997, having the highest methodological quality of the included studies, was also the only study to involve consumers in the development of the intervention. A recent Cochrane review (Nilsen 2006) which assessed the effects of consumer involvement on patient information material, found "moderate quality evidence that involving consumers in the development of patient information material results in material that is more relevant, readable and understandable to patients, without affecting their anxiety. This 'consumer-informed' material can also improve patients' knowledge". There is extensive research on patient/consumer involvement in research design (for example: Ali 2006; Guarino 2006; Kirkpatrick 2005; Oliver 2004; SCIE 2007; Thornton 2003); and of consumer perspectives on trial information presented during the consent process (for example: Featherstone 1998; Greenley 2006; Joffe 2001a; Robinson 2005; Stead 2005; Sugarman 1998). This seems a potentially fruitful avenue for improving research on the use of audio-visual interventions during the informed consent process. Given the narrow range of outcomes assessed by the included studies, it is also important that future evaluation of this and similar interventions take into account consumer views on relevant outcomes. Qualitative research on consumer perspectives may also usefully inform the development of interventions and selection of relevant outcomes.

\section{Quality of the evidence}

The methodological quality of included studies varied: only one study adequately concealed allocation to groups (Weston 1997); no study performed blinding or formally validated outcomes; yet three of the four studies attempted to minimise at least some potential sources of bias (Agre 2003b; Benson 1988; Weston 1997). From the current results, there appears to be no clear relationship between study findings and study quality - the two studies showing some clear positive effects of the video intervention on knowledge/ understanding were those assessed as at both lower (Weston 1997) 
and higher (Norris 1990) risks of bias related to methodological quality.

\section{Strengths and limitations of this review}

We have utilised the standard methods for Cochrane reviews, which are designed to minimise bias. Where possible, we contacted authors of included studies and key articles, and we thus obtained extensive information to supplement published trial reports. We have sought to ensure the wider applicability of the review by engaging consumer input throughout its development, and by consulting relevant consumer literature.

Despite these strengths, it is possible that we did not identify all relevant published or unpublished studies, particularly as informed consent trials are often published in very brief format in such journals as IRB: Ethics and Human Research. We welcome contact from anyone knowing of a potentially-eligible study not assessed for inclusion in this review. We did not conduct any handsearching, or any electronic searching of databases in languages other than English, although there were no language restrictions on the studies eligible for inclusion. The identified studies are all in English and conducted in higher-income countries, which may limit the generalisability of this review to other settings. We were unable to contact the authors of Norris 1990 and hence could only include limited information on this study.

Rate of participation or willingness to participate in a trial was a primary outcome specified for this review. We recognise however that this is a complex outcome that may be affected by a range of factors, such as knowledge and/or understanding, satisfaction, anxiety and others. In future updates of this review we will reexamine the selection and ordering of primary and secondary outcomes..

\section{Agreement and disagreements with other reviews or studies}

The findings of this review are consistent with other evidence about the effects of audio-visual interventions used in the informed consent process for potential trial participants. Previous studies have noted, for example, the relative scarcity of rigorous research on informed consent for research in comparison with that on informed consent for clinical treatment (Sugarman 1998). A recent systematic review of various interventions to improve trial participants' understanding of information disclosed during the informed consent process (Flory 2004) identified 12 video and computer multimedia interventions assessed in 9 studies (8 of which were randomised). Three of the four studies included in this review also appear in Flory's systematic review (Agre 2003b; Benson 1988; Weston 1997). Nine of the interventions resulted in no significant improvement in participants' understanding, although two of these did show improved retention of information at follow up.
Overall, the authors concluded that "multimedia and enhanced consent form interventions do not consistently improve research participants' understanding. Person-to-person interactions, especially the extended discussion interventions, may be more effective." Another recent review by Cohn 2007, which included a subset of those studies evaluated by Flory 2004, similarly concluded that no single approach (of simplified written consent forms, use of trained professionals and multimedia interventions) appeared universally effective.

\section{The impact of 'standard' information provision in informed consent research}

The underlying reasons for the variable effects of audio-visual and other multimedia interventions in this field are not well understood. As the importance of obtaining consent that is properly informed gains wider recognition, the control or standard information provision arms used in such research can operate as powerful interventions in their own right. This may make it more difficult to consistently detect an effect of audio-visual or other innovative informational interventions (Agre 2003a; Flory 2004). For example, while improvements in patients' understanding of clinical care have been shown with multimedia interventions, the same improvements are not consistently evident in the clinical research literature (Agre 1994; Flory 2004). Since the informed consent process for research typically involves formalised consent processes including written consent forms at a minimum, adding a novel multimedia intervention to existing consent procedures may achieve relatively little in terms of enhancing understanding, or other relevant outcomes, for potential trial participants (Flory 2004). Interestingly, both studies reporting improvements in knowledge/ understanding with audio-visual interventions in this review (Norris 1990; Weston 1997) incorporated written information and discussions with the study nurse in their standard consent procedures, which could be regarded as powerful controls. The impact of standard informed consent procedures does not seem to account for the pattern of results in this review but its influence remains an important consideration for future trials.

\section{Issues in achieving informed consent}

Many previous reviews and studies have noted the relatively poor levels of knowledge/understanding of potential trial participants in relation to the informed consent process (for example: Campbell 2004; Cohn 2007; Dunn 2006; Guarino 2006; Hietanen 2000; Harth 1995; Lavori 1999; Robinson 2005; Verástegui 2006). These problems have persisted despite the use of a range of interventions, among them multimedia interventions, designed specifically to enhance this process and to promote consent that is properly informed (Cohn 2007; Flory 2004; Robinson 2005). In this review, participants did not consistently achieve $100 \%$ correct responses on knowledge questionnaires even when studies reported an increase in knowledge/understanding following audio-visual 
interventions. Therefore, even those participants whom we might regard as 'successful' recipients of the intervention may only partially understand the elements required for consent that is informed. The educational levels of participants included in this review tended to be high; previous research has indicated that higher education levels, together with other factors such as younger age and better reading levels, consistently predict better knowledge of informed consent information (Agre 2003a). The converse also seems true: fewer years of formal education and older age are both associated with poorer understanding of consent information (Flory 2004; Joffe 2001a; Sugarman 1998). There may also be differential effects on recalled information for different informed consent information formats by people with lower, compared with higher, reading levels (Campbell 2004).

Research on informed consent in low-and-middle income countries has also highlighted several predictors of poor understanding of clinical trial information. These include poor living conditions, limited literacy or lack of education, and the complexity of information provided in the informed consent process (Joseph 2006; Verástegui 2006). Other studies have suggested that miscomprehension of information about clinical trials, particularly the more complex concepts such as randomisation, placebo, withdrawal and side effects, may reach very high levels among potential participants in developing countries, and that these levels are substantially higher than those in industrialised countries (Krosin 2006; Moodley 2005; Verástegui 2006). People in developing countries may also have few opportunities to receive health care outside clinical trials and this may influence their decision to consent, even where their comprehension or understanding of the trial is low (Krosin 2006; Verástegui 2006). Such research suggests that predictors of informed consent are complex; are influenced by social, cultural and economic factors; and may not readily generalise from industrialised to developing countries. This review does not clarify the effects of audio-visual interventions on outcomes for people in low-and-middle income countries, and this remains an important gap in research.

Outcome assessment for informed consent for research is problematic. Even for a seemingly uncomplicated outcome like knowledge/understanding, for example, there is not a 'gold standard' assessment method (Cohn 2007; Flory 2004). What constitutes 'adequate' knowledge or understanding with regard to clinical trial participation remains contentious (Stead 2005). For example, is a certain percentage of correct responses considered adequate? Where does this threshold lie? Is understanding of some components essential while of others less so? Is there agreement between researchers and participants in terms of what is most important? (Agre 2003a; Dunn 2006; Guarino 2006; Joffe 2001b; Robinson 2005). These issues require further research.

Understanding or comprehension is, however, only one of several components of meaningful informed consent (Sugarman 1998). Researchers and ethicists refer to 'decisional capacity' - an individual's ability to use information when deciding whether to under- take a particular treatment or to participate in research - to describe how meaningful the informed consent for clinical treatment or research is (Dunn 2006). Decisional capacity includes at least four domains: understanding or comprehension of the relevant information; appreciation of the significance of the information and application to an individual's own situation; the ability to use the information in decision-making; and the ability to express a consistent choice or decision (Dunn 2006; Palmer 2005). Each of these components need to be addressed for consent to be well informed. In a recent systematic review of tools to assess decisional capacity for both treatment and research, Dunn and colleagues noted that only a minority of tools assessed all four domains of decisional capacity, with most focussing solely or primarily on understanding or comprehension (Dunn 2006). A few tools assessing decisional capacity more comprehensively, such as the validated MacArthur Competence Assessment Tool for Clinical Research, were identified, however the authors noted that clear consensus and definition of the range of relevant outcomes is required in informed consent research (Dunn 2006; Sugarman 1998).

Research on the informed consent process for research purposes is a complex area. Ensuring that consent for treatment is informed is complicated enough. Informed consent for research must also aim to ensure that participants comprehend and then recall complex information about trial design and conduct that may be wholly unfamiliar to them (Robinson 2005; Sugarman 1998). Limited health literacy is only one of the barriers to achieving informed consent (Stead 2005): even providing clear, accurate and comprehensive information to potential participants may not ensure that they interpret complex research concepts consistently (Featherstone 1998; Robinson 2005; Stead 2005). Instead it appears that people try to make sense of the complex technical concepts associated with research by interpreting these concepts in (more or less accurate) ways that best make sense to them, or which fit with their values and prior knowledge (Featherstone 1998; Robinson 2005; Stead 2005). Other characteristics of research itself can present further barriers to informed consent. These include the expectations that people have about usual clinical treatment; whether participants and researchers have a shared understanding of research terminology; and whether participants are able to understand and to recall the different aims of clinical care and research (Featherstone 1998; Joffe 2001a; Robinson 2005; Sugarman 1998).

Those designing and implementing interventions, such as audiovisual interventions, to enhance the informed consent process for research, need to consider whether such interventions should be prepared and delivered in a generic manner to all recipients (Sugarman 1998). This review supports previous studies which have indicated that no single intervention format appears consistently effective or superior to other formats for all participants (Agre 2003a; Cohn 2007; Flory 2004). Other research has suggested that decisional capacity varies considerably from person to person, and cannot be predicted only on the basis of clinical diagnosis (Palmer 2005). The mixed results in this review might re- 
flect differences in the populations included in individual studies; or may reflect other differences that cannot be identified clearly from existing research at this time. What is clear is that research has consistently shown poor or imperfect knowledge/understanding, and other outcomes, following blanket delivery of interventions to improve informed consent for potential research participants. Considering individuals' decisional capacity and tailoring the informed consent information appropriately might be one approach to improving informed consent and ensuring that consent is meaningful (Palmer 2005; Sugarman 1998). In related areas such as patient information materials, decision aids and other interventions to help people participate effectively in health care and decision making, the tailoring of information to individuals has been identified as a potential way forward. A recent study piloting the effects of a decision aid in the informed consent process for recruitment to a breast cancer trial, for example, also reported on the effectiveness of this intervention in improving understanding and other relevant outcomes among potential trial participants (Juraskova 2007). The adaptation of this approach to audio-visual formats might also be a promising avenue to explore with respect to supporting informed decision-making and consent for potential trial participants.

\section{AUTHORS' CONCLUSIONS}

\section{Implications for practice}

The value of audio-visual interventions as a tool for helping to improve the informed consent process for people considering participating in clinical trials remains unclear and requires additional research. Evidence is mixed as to whether audio-visual interventions enhance people's knowledge of the trial they are considering joining, and/or the health condition the trial is designed to address; one study showed improved retention of knowledge amongst intervention recipients. The intervention may also have small positive effects on the quality of information disclosed, and willingness to participate; however again the evidence is weak. There is at present no evidence of harm, although a range of important outcomes, such as anxiety, were not assessed. In the absence of clear results, triallists should continue to explore innovative methods of providing information to potential trial participants during the informed consent process, and should consider tailoring the interventions to particular participant groups.

\section{Implications for research}

The effects of audio-visual interventions for use in the informed consent process for trials remain unclear: four small trials, each with methodological limitations, reported mixed effects on a small range of outcomes. Further rigorous research is needed. Future studies should conduct content assessment of video and other innovative informational interventions for people at differing levels of understanding and education; also for different age and ethnic groups, in different settings and countries. Offering individual participants a choice of formats for informed consent information should also be evaluated; as should tailored interventions for use in the informed consent process for different participant groups.

Triallists should investigate which delivery components of an audio-visual intervention for informed consent make it more or less effective, systematically assessing the effects of different elements such as timing, provider and duration. Ideally, consumers should be actively involved in developing the intervention.

Future studies should include adequate outcome measurement, including the validation and reliability testing of all outcome measures. Studies should assess a range of outcomes in order to assess decisional capacity of potential trial participants, preferably using validated measures of informed consent, and including the consumer-oriented outcomes that were not assessed by included studies, such as: participant (or guardian) satisfaction with 1) information provided about the clinical study, 2) the media used to convey the information, and 3) the decision making process; anxiety; adherence with the study protocol; and withdrawal from the subsequent study following consent.

Triallists should seek to minimise the risk of bias in their studies by ensuring adequate randomisation and allocation concealment, and by blinding (where possible) participants, intervention providers and outcome assessors. Study reporting in this field also requires improvement; trial authors should adhere to the recommendations of the CONSORT statement (www.consort-statement.org), particularly in terms of the methods they use to minimise the risk of bias, and in terms of participant numbers and the flow of participants through each stage of the trial.

\section{ACKNOWLEDGEMENTS}

For their input to the protocol for this review, we are grateful to Clarissa Cook, Dell Horey, and Jane Putsey.

We thank Annie Brindley and Caroline Crowther for their contributions as co-authors to the protocol for this review, and Caroline for her assistance during the early stages of the review.

We have been assisted immeasurably by trial authors who provided information, assistance and additional papers, and by other experts in the field who provided additional papers and contacts with other experts. We are particularly grateful to the following authors who provided large amounts of detailed information that assisted us in completing this review: Julie Weston (first author), for information provision; also Donna Wirshing for providing an additional full-text paper and further information; Patricia Agre (additional information about studies, helped with contact details of other authors); Greg Sachs (additional papers, contact details 
for other authors and researchers in the field); Laura Dunn for providing further details about studies considered for inclusion in this review; and to Nancy Kass for providing additional information about the publication of an ongoing trial, characteristics of this trial and further references.

We are grateful to the staff and editors of the Cochrane Consumers and Communication Review Group, particularly to Sandy Oliver and Kelly Allen; to Shirley Ward and Judith Stoelwinder (current and past Trials Search Coordinators) for the design and running of searches and retrieval of references, and to external peer reviewers (including consumer reviewers) for their invaluable feedback.

\section{R E F E R E N C E S}

\section{References to studies included in this review}

Agre 2003b \{published and unpublished data\}

Agre P, Rapkin B. Improving informed consent: a comparison of four consent tools. IRB: Ethics and Human Research 2003;25(5):1-7.

Benson 1988 \{published and unpublished data\} Benson PR, Appelbaum PS, Lidz CW, Winslade WJ. Information disclosure, subject understanding, and informed consent in psychiatric research. Law and Human Behavior 1988;12:455-75.

Norris 1990 \{published data only\} Norris DR, Phillips MR. Using instructive videotapes to increase patient comprehension of informed consent. Journal of Clinical Research \& Pharmacoepidemiology 1990;4 (4):263-8. [MEDLINE: yes]

Weston 1997 \{published and unpublished data\} Weston J, Downes J, Hannah M. Evaluating the benefits of patient video during the informed consent process. Controlled Clinical Trials. 1995; Vol. 16:73S (abstract). * Weston J, Hannah M, Downes J. Evaluating the benefits of a patient information video during the informed consent process. Patient Education \& Counseling 1997;30(3): 239-45. [MEDLINE: yes]

\section{References to studies excluded from this review}

Agre 1994 \{published data only\}

Agre P, Kurtz RC, Krauss B. A randomised trial using videotape to present consent information for colonoscopy. Gastrointestinal Endoscopy 1994;40:271-6.

Agre 2002 \{published data only\}

Agre P, Rapkin B, Dougherty J, Wilson R. Barriers encountered conducting informed consent research. IRB: Ethics and Human Research 2002;24(4):1-5.

Agre 2003a \{published and unpublished data\} Agre P, Campbell FA, Goldman BD, Boccia ML, Kass $\mathrm{N}$, McCullough LB, et al.Improving informed consent: the medium is not the message. IRB: Ethics and Human Research (Supplement) 2003;25(5):S11-19.
Barbour 1978 \{published data only\}

Barbour GL, Blumenkrantz MJ. Videotape aids informed consent decision. JAMA 1978;240(25):2741-2.

[MEDLINE: yes]

Benitez 2002 \{published data only\}

Benitez O, Devaux D, Dausset J. Audiovisual

documentation of oral consent: A new method of informed consent for illiterate populations. Lancet 2002;359(9315):

1406-7. [MEDLINE: yes]

Benson 1985 \{published data only\}

Benson PR, Roth LH, Winslade WJ. Informed consent in psychiatric research: preliminary findings from an ongoing investigation. Social Science \& Medicine. 1985;20(12): 1331-41. [MEDLINE: yes]

Brandon 1991 \{published data only\} Brandon S. Ethics, economics and science. Journal of the Royal Society of Medicine. 1991;84(10):575-7. [MEDLINE: ?]

Brown 2004 \{published data only\}

Brown RF, Butow PN, Butt DG, Moore AR, Tattersall MH. Developing ethical strategies to assist oncologists in seeking informed consent to cancer clinical trials. Social Science \& Medicine. 2004;58(2):379-90. [MEDLINE: yes? - need to see full paper]

Campbell 2004 \{published data only\} Campbell FA, Goldman BD, Boccia ML, Skinner M. The effect of format modifications and reading comprehension on recall of informed consent information by low-income parents: a comparison of print, video, and computer-based presentations. Patient Education and Counseling 2004;53: 205-16.

Curbow 2004 \{published data only\}

Curbow B, Fogarty LA, McDonnell K, Chill J, Scott LB.

Can a brief video intervention improve breast cancer clinical trial knowledge and beliefs?. Social Science \& Medicine 2004;58(1):193-205. [MEDLINE: yes]

Dunbar 1989 \{published data only\}

Anonymous. Implementation of a multicomponent process to obtain informed consent in the Diabetes Control 
and Complications Trial. The DCCT Research Group. Controlled Clinical Trials 1989;10:83-96.

Dunbar J, Cleary PA, Siebert C, Baker L, Brink S, Nathan DM. Implementation of a multicomponent process to obtain informed consent in the diabetes control and complications trial. Controlled Clinical Trials 1989;10(1): 83-96. [MEDLINE: ?]

Dunn 2001 \{published and unpublished data\} Dunn LB, Lindamer LA, Palmer BW, Schneidermann LJ, Jeste DV. Enhancing comprehension of consent for research in older patients with psychosis: a randomised study of a novel consent procedure. American Journal of Psychiatry 2001;158(11):1911-3.

\section{Fisher 1991 \{published data only\}}

Fisher WB, et al.Clinical trials in cancer therapy: efforts to improve patient enrollment by community oncologists. Medical \& Pediatric Oncology 1991;19:165-8. [MEDLINE: yes - useful for literature review, but not really trial]

Flory 2004 \{published data only\}

Flory J, Emanuel E. Interventions to improve research participants' understanding in informed consent for research: a systematic review. JAMA 2004;292(13): 1593-601.

Fureman 1997 \{published data only\}

Fureman I, Meyers K, McLellan AT, Metzger D, Woody G. Evaluation of a video-supplement to informed consent: injection drug users and preventive HIV vaccine efficacy trials. AIDS Education \& Prevention 1997;9(4):330-41. [MEDLINE: yes]

Hall 2001 \{published data only\} Hall JM, Stevens PE, Pletsch PK. Team research using qualitative methods: investigating children's involvement in clinical research. Journal of Family Nursing 2001;7(1):7-31. [MEDLINE: ?]

Harzstark 2001 \{published data only\} Harzstark AL, Schurman CR, Luce J, Carlson RW. Studying the effectiveness of videos in conveying medical information regarding breast cancer risks and the Study of Tamoxifen and Raloxifene (STAR) trial. Breast Cancer Research \& Treatment 2001;69:268.

Hopper 1994 \{published data only\} Hopper KD, Zajdel M, Hulse SF, Yoanidis NR, TenHave TR, Labuski MR, et al.Interactive method of informing patients of the risks of intravenous contrast media. Radiology 1994;192(1):67-71. [MEDLINE: ?]

Hougham 2003 \{published data only\} Hougham GW, Sachs GA, Danner D, Mintz J, Patterson M, Roberts LW, et al.Empirical research on informed consent with the cognitively impaired. IRB: Human Ethics and Research (Supplement) 2003;25(5):1-7.

Jefford 2002 \{published data only\} Jefford M, Tattersall MHN. Informing and involving cancer patients in their own care. Lancet Oncology 2002;3(10): 629-37. [MEDLINE: ?]
Jensen 1993 \{published data only\}

Jensen AB, Madsen B, Andersen P, Rose C. Information for cancer patients entering a clinical trial - an evaluation of an information strategy. European Journal of Cancer 1993;29A (16):2235-8.

Jepson 2001 \{published data only\} Jepson RG, Forbes CA, Sowden AJ, Lewis RA. Increasing informed uptake and non-uptake of screening: evidence from a systematic review. Health Expectations 2001;4(2): 116-26. [MEDLINE: ?]

Jimison 1998 \{published data only\} Jimison HB, Sher PP, Appleyard R, LeVernois Y. The use of multimedia in the informed consent process. Journal of the American Medical Informatics Association 1998;5(3): 245-56. [MEDLINE: yes]

Joseph 2006 \{published data only\} Joseph P, Schakman BR, Horwitz R, Nerette S, Verdier RI, Dorsainvil $\mathrm{D}$, et al.The use of an educational video during informed consent in an HIV clinical trial in Haiti. Journal of Acquired Immune Deficiency Syndromes 2006;42(5):588-91.

Koh 2001 \{published data only\} Koh TH, Collie L, Budge D, Butow P. Informed consent in neonatal randomised trials. Lancet. 2001;357(9266): 1445-6. [MEDLINE: yes - need full paper to check]

\section{Krogh 1999 \{published data only\}}

Krogh KS, Lindsay PH. Including people with disabilities in research: implications for the field of augmentative and alternative communication. AAC: Augmentative \& Alternative Communication 1999;15(4):222-33. [MEDLINE: yes - maybe?]

Krouse 2001 \{published data only\} Krouse HJ. Video modelling to educate patients. Journal of Advanced Nursing. 2001;33(6):748-57. [MEDLINE: ?]

Lenert 2000 \{published data only\} Lenert LA, Ziegler J, Lee T, Unfred C, Mahmoud R. The risks of multimedia methods: effects of actor's race and gender on preferences for health states. Journal of the American Medical Informatics Association 2000;7(2):177-85. [MEDLINE: ?]

Llewellyn 1995 \{published data only\} Llewellyn Thomas HA, Thiel EC, Sem FWC, Harrison Woermke DE. Presenting clinical trial information: a comparison of methods. Patient Education \& Counseling 1995;25:97-107. [MEDLINE: yes]

Maslin 1998 \{published data only\} Maslin AM, Baum M, Walker JS, A'Hern R, Prouse A. Using an interactive video disk in breast cancer patient support. Nursing Times 1998;94:52-5. [MEDLINE: ?]

McPherson 2001 \{published data only\} McPherson CJ, Higginson IJ, Hearn J. Effective methods of giving information in cancer: a systematic literature review of randomized controlled trials. Journal of Public Health Medicine. 2001;23(3):227-34. [MEDLINE: ?] 
McPherson 2002 \{published data only\}

McPherson EC, Ray SC, Rieser PA, Manasco PK.

Pharmacogenetic research: perceptions of informed consent. Drug Information Journal 2002;36(1):83-93. [MEDLINE: yes]

McTiernan 1995 \{published data only\} McTiernan A, Rossouw J, Manson J, Franzi C, Taylor V, Carleton R, et al.Informed consent in the women's health initiative clinical trial and observational study. Journal of Women's Health 1995;4(5):519-29. [MEDLINE: yes]

Mittal 2007 \{published data only\}

Mittal D, Palmer B, Dunn L, Landes R, Ghormley C, Beck $\mathrm{C}$, et al.Comparison of two enhanced consent procedures for patients with mild Alzheimer disease or mild cognitive impairment. American Journal of Geriatric Psychiatry 2007; 15(2):163-7.

Morrow 1978 \{published data only\}

Morrow G, Gootnick J, Schmale A. A simple technique for increasing cancer patients' knowledge of informed consent to treatment. Cancer 1978;42:793-9.

Moseley 2006 \{published data only\} Moseley TH, Wiggins MN, O'Sullivan P. Effects of presentation method on the understanding of informed consent. British Journal of Opthalmology 2006;90:990-3.

Palladino 2002 \{published data only\} Palladino ML. Challenges in the informed consent process: identifying design strategies that enhance communication in adult clinical trials. Research Practitioner 2002;3(5):164-71. [MEDLINE: yes]

Pignone 2000 \{published data only\} Pignone M, Harris R, Kinsinger L. Videotape-based decision aid for colon cancer screening: a randomized, controlled trial. Annals of Internal Medicine 2000;133(10): 761-9. [MEDLINE: ?]

Pletsch 2001a \{published data only\} Pletsch PK, Stevens PE. Inclusion of children in clinical research: lessons learned from mothers of diabetic children. Clinical Nursing Research 2001;10(2):140-62. [MEDLINE: ?]

Pletsch 2001b \{published data only\}

Pletsch PK, Doerr B, Irwin D. Focus group interviews: a method for creating partnerships with African-American women in the design of research informed consent. Journal of National Black Nurses Association 2001;12(1):42-7. [MEDLINE: yes]

Pletsch 2001c \{published data only\}

Pletsch PK, Stevens PE. Children in research: informed consent and critical factors affecting mothers. Journal of Family Nursing 2001;7(1):50-70. [MEDLINE: ?]

Rangel 2002 \{published data only\}

Rangel SJ, Narasimhan B, Geraghty N, Moss RL. Development of an Internet-based protocol to facilitate randomized clinical trials in pediatric surgery. Journal of Pediatric Surgery 2002;37(7):990-3. [MEDLINE: yes]
Ruckdeschel 1996 \{published data only\}

Ruckdeschel JC, Albrecht TL, Blanchard C, Hemmick RM. Communication, accrual to clinical trials, and the physician-patient relationship: implications for training programs. Journal of Cancer Education 1996;11(2):73-9. [MEDLINE: ?]

Sachs 2003 \{published data only\} Sachs G, Hougham GW, Sugarman J, Agre P, Broome ME, Geller $\mathrm{G}$, et al.Conducting empirical research on informed consent: challenges and questions. IRB: Ethics and Human Research (Supplement) 2003;25(5):S4-10.

Sheldon 1993 \{published data only\} Sheldon JM, Fetting JH, Siminoff LA. Offering the option of randomized clinical trials to cancer patients who overestimate their prognoses with standard therapies. Cancer Investigation 1993;11(1):57-62. [MEDLINE: ?]

Shurnas 2003 \{published data only\}

Shurnas PS, Coughlin MJ. Recall of the risks of forefoot surgery after informed consent. Foot \& Ankle International. 2003;24(12):904-8. [MEDLINE: ?]

Simes 1986 \{published data only\}

Simes J, Tattersall MHN, Coate AS, Raghavan D, Solomon HJ, Smartt H. Randomised comparison of procedures for obtaining informed consent in clinical trials of treatment for cancer. BMJ 1986;293:1065-8.

Sugarman 1998 \{published data only\}

Sugarman J, McCrory DC, Hubal RC. Getting meaningful informed consent from older adults: a structured literature review of empirical research. Journal of the American Geriatrics Society 1998;46:517-24. [MEDLINE: yes]

Thomas 2000 \{published data only\}

Thomas R, Daly M, Perryman B, Stockton D. Forewarned is forearmed: benefits of preparatory information on video cassette for patients receiving chemotherapy or radiotherapy, a randomised controlled trial. European Journal of Cancer 2000;36(12):1536-43. [MEDLINE: yes]

Thornton 1993 \{published data only\} Thornton H. Learning about randomised controlled trials. Bulletin of Medical Ethics. 1993;87:21-3. [MEDLINE: ?]

Tindall 1994 \{published data only\} Tindall B, Forde S, Ross MW, Goldstein D, Barker S, Cooper DA. Effects of two formats of informed consent on knowledge among persons with advanced HIV disease in a clinical trial of didanosine. Patient Education and Counseling 1994;24:261-6.

Tymchuk 1986 \{published data only\} Tymchuk AJ, Ouslander JG, Rader N. Informing the elderly, a comparison of four methods. Journal of the American Geriatrics Society 1986;34:818-22.

Varnhagen 2005 \{published data only\} Varnhagen CK, Gushta M, Daniels J, Peters TC, Law D, Hirsch R, et al.How informed is online informed consent?. Ethics and Behaviour 2005;15(1):37-48. 
Ventura 2003 \{published data only\}

Ventura J, Wirshing D, Kern R, Mintz J, Appelbaum P.

Decision making and informed consent in schizophrenia.

Biological Psychiatry. 2003; Vol. 53:1S-217S.

Westreich 1995 \{published data only\}

Westreich L, Levine S, Ginsburg P, Wilets I. Patient

knowledge about electroconvulsive therapy: effect of an

informational video. Convulsive Therapy 1995;11(1):32-7.

Wirshing 2005 \{published data only\}

Mintz J, Wirshing DA, et al.cited in Agre et al (2003).

Wirshing DA, Mintz J, Kern R, Boyd J. A videotape intervention to enhance the informed consent process for patients participating in clinical research trial. Schizophrenia Research 2003;60:306

* Wirshing DA, Sergi MJ, Mintz J. A videotape intervention to enhance the informed consent process for medical and psychiatric treatment research. American Journal of Psychiatry 2005;162(1):186-8.

Wragg 2000 \{published data only\}

Wragg JA, Robinson EJ, Lilford RJ. Information presentation and decisions to enter clinical trials: a hypothetical trial of hormone replacement therapy. Social Science \& Medicine 2000;51(3):453-62. [MEDLINE: yes]

\section{References to studies awaiting assessment}

Hougham 2003b \{published data only\}

Hougham GW, Sachs GA, Danner D, Mintz J, Patterson M, Roberts L, et al.University of California at Los Angeles study. Cited in Hougham 2003: study not cited by name, authors, only by affiliation.

Kass \{published data only\}

Kass N, Sugarman J, Taylor H, Fogarty L, Daugherty C, Carducci $\mathrm{M}$, et al.Improving understanding in early phase cancer trials. [Cited in Agre 2003 and Flory 2004].

\section{Additional references}

\section{Ali 2006}

Ali K, Roffe C, Crome P. What patients want: consumer involvement in the design of a randomised controlled trial of routine oxygen supplementation after acute stroke. Stroke 2006;37:865-71.

Ballard 2004

Ballard HO, Shook LA, Desai NS, Anand KJ. Neonatal research and the validity of informed consent obtained in the perinatal period.. Journal of Perinatology 2004;24(7): $409-15$.

Brown 2006

Brown P, Brunnhuber K, Chalkidou K, Chalmers I, Clarke M, Fenton M, et al.How to formulate research recommendations. BMJ 2006;333:804-6.

\section{CCRG 2004}

Cochrane Consumers and Communication Review Group. Data extraction template for Cochrane reviews. http:// www.latrobe.edu.au/cochrane/assets/downloads/DET.doc (accessed July 2004) 2004 (version 1.1.1).

\section{Chappuy 2006}

Chappuy H, Doz F, Blanche S, Gentet JC, Pons G, Treluyer JM. Parental consent in paediatric clinical research. Archives of Disease in Childhood 2006;91(2):112-6.

Clarke 2001

Clarke M, Oxman AD, editors. Cochrane Reviewers' Handbook 4.1.4 [updated October 2001]. The Cochrane Library. Oxford: Update Software, 2001, issue Issue 4.

\section{Cohn 2007}

Cohn E, Larson E. Improving participant comprehension in the informed consent process. Journal of Nursing Scholarship 2007;39(3):273-80.

\section{Dresden 2001}

Dresden GM, Levitt MA. Modifying standard industry clinical trial consent form improves patient information retention as part of the informed consent process. Academic Emergency Medicine 2001;8(3):246-52.

Dunn 2001a

Dunn LB, Lindamer LA, Schneiderman LJ, Jeste DV. A novel, computer-based enhancement of informed consent in older patients with psychotic disorders. 14th Annual Meeting of the American Association for Geriatric Psychiatry. San Francisco, CA, USA, 2001 23rd-26th February.

\section{Dunn 2006}

Dunn LB, Nowrangi MA, Palmer BW, Jeste DV, Saks ER. Assessing decisional capacity for clinical research or treatment: a review of instruments. American Journal of Psychiatry 2006;163(8):1323-34.

\section{Edwards 2006}

Edwards AGK, Evans R, Dundon J, Haigh S, Hood K, Elwyn GJ. Personalised risk communication for informed decision making about taking screening tests. Cochrane Database of Systematic Reviews 2006, Issue 4. [Art. No.: CD001865. DOI: 10.1002/14651858.CD001865.pub2]

\section{Featherstone 1998}

Featherstone K, Donovan JL. Random allocation or allocation at random? Patients' perspectives of paticipation in a randomised controlled trial. BMJ 1998;317:1177-80.

Franck 2007

Franck LS, Winter I, Oulton K. The quality of parental consent for research with children: a prospective repeated mesure self-report survey. International Journal of Nursing Studies 2007;44(4):525-33.

\section{Greenley 2006}

Greenley RN, Drotar D, Zyzanski SJ, Kodish E. Stability of parental understanding of random assignment in childhood leukemia trials: an empirical examination of informed consent. Journal of Clinical Oncology 2006;24(6):891-7.

Guarino 2006

Guarino P, Elbourne D, Carpenter J, Peduzzi P. Consumer involvement in consent document development: a multicenter cluster randomized trial to assess study particpants' understanding. Clinical Trials 2006;3:19-30. 


\section{Harth 1995}

Harth SC, Thong YH. Parental perceptions and attitudes about informed consent in clinical research involving children. Social Science Medicine 1995;41 (12):1647-51.

\section{Hietanen 2000}

Hietanen P, Aro AR, Holli K, Absetz P. Information and communication in the context of a clinical trial. European Journal of Cancer 2000;36:2096-2104.

\section{Higgins 2005}

Higgins JPT, Green S, editors. Cochrane Handbook for Systematic Reviews of Interventions 4.2.5 [updated May 2005]. The Cochrane Library. Chichester, UK: John Wliey \& Sons Ltd, 2005.

\section{Joffe 2001a}

Joffe S, Cook EF, Cleary PD, Clark JW, Weeks JC. Quality of informed consent in cancer clinical trials: a crosssectional survey. Lancet 2001;358:1772-7.

\section{Joffe 2001b}

Joffe S, Cook EF, Cleary PD, Clark JW, Weeks JC. Quality of informed consent: a new measure of understanding among research subjects. Journal of the National Cancer Institute 2001;93(2):139-47.

\section{Juraskova 2007}

Juraskova I, Butow P, Lopez AL, Seccombe M, Boyle F, McCarthy N, et al.Improving informed consent in clinical trials: successful piloting of a decision aid. Journal of Clinical Oncology 2007;25(11):1443-4.

\section{Kirkpatrick 2005}

Kirkpatrick CMJ, Roughead EE, Monteith GR, Tett SE. Consumer involvement in Quality Use of Medicines (QUM) projects - lessons from Australia. BMC Health Services Research 2005;5:75.

\section{Krosin 2006}

Krosin MT, Klitzman R, Levin B, Cheng J, Ranney ML. Problems in comprehension of informed consent in rural and peri-urban Mali, West Africa. Clinical Trials 2006;3(3): 306-13.

\section{Kruse 2000}

Kruse AY, Kjaergard LL, Krogsgaard K, Gluud C, Mortensen EL, Gottschau A, Bjerg AM. A randomized trial assessing the impact of written information on outpatients' knowlege about and attitude towards randomized clinical trials. Controlled Clinical Trials 2000;21(3):223-40.

\section{Kupst 2003}

Kupst MJ, Patenaude AF, Walco GA, Sterling C. Clinical trials in pediatric cancer: parental perspectives on informed consent. Journal of Pediatric Hematology and Oncolology 2003;25(10):787-90.

\section{Lavori 1999}

Lavori PW, Sugarman J, Hays MT, Feussner JR. Improving informed consent in clinical trials: a duty to experiment. Controlled Clinical Trials 1999;20:187-193.
Mapstone 2007

Mapstone J, Elbourne D, Roberts I. Strategies to improve recruitment to research studies. Cochrane Database of Systematic Reviews 2007, Issue 2.

Miller 2005

Miller VA, Drotar D, Burant C, Kodish E. Clinician-parent communication during informed consent for pediatric leukemia trials. Journal of Pediatric Psychology 2005;30(3): 219-29.

\section{Moodley 2005}

Moodley K, Pather M, Myer L. Informed consent and participant perceptions of influenza vaccine trials in South Africa. Journal of Medical Ethics 2005;31(12):727-32.

Nilsen 2006

Nilsen ES, Myrhaug HT, Johansen M, Oliver S, Oxman AD. Methods of consumer involvement in developing healthcare policy and research, clinical practice guidelines and patient information material. Cochrane Database of Systematic Reviews 2006, Issue 3. [Art. No.: CD004563. DOI: 10.1002/14651858.CD004563.pub2]

\section{O'Connor 2003}

O'Connor AM, Stacey D, Entwistle V, Llewellyn-Thomas H, Rovner D, Holmes-Rovner M, Tait V, et al.Decision aids for people facing health treatment or screening decisions. Cochrane Database of Systematic Reviews 2003, Issue 1.[Art. No.: CD001431. DOI: 10.1002/ 14651858.CD001431.pub2]

\section{Oliver 2004}

Oliver S, Clarke-Jones L, Rees R, Milne R, Buchanan P, Gabbay J, et al.Involving consumers in research and development agenda setting for the NHS: developing an evidence-based approach. Health Technology Assessment 2004; Vol. 8, issue 15.

\section{Palmer 2005}

Palmer BW, Dunn LB, Appelbaum PS, Mudaliar S, Thal L, Henry R, et al.Assessment of capacity to consent to research among older persons with schizophrenia, Alzheimer's disease or diabetes mellitus. Archives of General Psychiatry 2005;62: 726-33.

Pope 2003

Pope JE, Tingey DP, Arnold JM, Hong P, Ouimet JM, Krizova A. Are subjects satisfied with the informed consent process? A survey of research participants. Journal of Rheumatology 2003;30(4):815-24.

\section{Raffle 2001}

Raffle A. Information about screening - is it to achieve high uptake or to ensure informed choice?. Health Expectations 2001;4:92-8

\section{Rendell 2007}

Rendell JM, Merritt RK, Geddes JR. Incentives and disincentives to participation by clinicians in randomised controlled trials. Cochrane Database of Systematic Reviews 2007, Issue 2.[Art. No.: MR000021. DOI: 10.1002/ 14651858.MR000021.pub3] 
Robinson 2005

Robinson EJ, Kerr CEP, Stevens AJ, Lilford RJ, Braunholtz DA, Edwards SJ. Lay public's understanding of equipoise and randomisation in randomised controlled trials. Health Technology Assessment 2005; Vol. 9, issue 8.

Ruccione 1991

Ruccione K, Kramer RF, Moore IK, Perin G. Informed consent for treatment of childhood cancer: factors affecting parents' decision making. Journal of Pediatric Oncology Nursing 1991;8(3):112-21.

\section{Ryan 2007}

Ryan R, Hill S, Broclain D, Horey D, Oliver S, Prictor M. Cochrane Consumers and Communication Review Group: Study Quality Guide. http://www.latrobe.edu.au/cochrane/ resources.html (last accessed 5 Sep 2007) 2007.

\section{Schats 2003}

Schats R, Brilstra EH, Rinkel GJ, Algra A, Van Gijn J. Informed consent in trials for neurological emergencies: the example of subarachnoid haemorrhage. Journal of Neurology Neurosurgery and Psychiatry 2003;74(7):988-91.

SCIE 2007

Social Care Institute for Excellence (SCIE). Research resource 02: Collection of examples of service user and carer participation in systematic reviews. http://www.scie.org.uk/ publications/researchresources/rr02.asp (accessed 5 Sep 2007) February 2007.

\section{Simon 2001}

Simon C, Eder M, Raiz P, Zyzanski S, Pentz R, Kodish ED. Informed consent for pediatric leukemia research: clinician perspectives. Cancer 2001;92(3):691-0.

Stanley 1998

Stanley BM, Walters DJ, Maddern GJ. Informed consent: how much information is enough?. Australian and New Zealand Journal of Surgery 1998;68(11):788-91.

\section{Stead 2005}

Stead M, Eadie D, Gordon D, Angus K. "Hello hello it's English I speak!”: a qualitative exploration of patients' understanding of the science of clinical trials. Journal of Medical Ethics 2005;31:664-9.

\section{Stryker 2006}

Stryker JE, Wray RJ, Emmons KM, Winer E, Demetri G. Understanding the decisions of cancer clinical trial participants to enter research studies: factors associated with informed consent, patient satisfaction, and decisional regret. Patient Education and Counselling 2006;63(1-2):104-9.

\section{Tait 2003}

Tait AR, Voepel-Lewis T, Malviya S. Do they understand? (part I): parental consent for children participating in clinical anesthesia and surgery research. Anesthesiology 2003; 98(3):603-8.

\section{Thomas 1999}

Thomas R, Deary A, Kaminiski E, Stockton D, De Zueew N. Patients' preferences for video cassette recorded information: effect of age, sex and ethnic group. European Journal of Cancer Care 1999;8:83-6.

\section{Thornton 2003}

Thornton H, Edwards A, Elwyn G. Evolving the multiple roles of 'patients' in health-care research: reflections after involvement in a trial of shared decision-making. Health Expectations 2003;6:189-97.

\section{Verástegui 2006}

Verástegui EL. Consenting of the vulnerable: the informed consent procedure in advanced cancer patients in Mexico. BMC Medical Ethics 2006;7:E13.

\section{Wilets 2005}

Wilets I, Schears RM, Gligorov N. Communicating with subjects: special challenges for resuscitation research. Academic Emergency Medicine 2005;12:1060-3.

\section{Williams 2003}

Williams, BF, French, JK, White. HD. Informed consent during the clinical emergency of acute myocardial infarction (HERO-2 consent substudy): a prospective observational study. Lancet 2003;361(9361):918-22.

\section{WMA 2000}

World Medical Association Declaration of Helsinki. Ethical Principles for Medical Research Involving Human Subjects. Adopted by the 18th WMA General Assembly Helsinki, Finland, June 1964 and amended by the 29th WMA General Assembly, Tokyo, Japan, October 1975, 35th WMA General Assembly, Venice, Italy, October 1983, 41st WMA General Assembly, Hong Kong, September 1989, 48th WMA General Assembly, Somerset West, Republic of South Africa, October 1996 and 52nd WMA General Assembly, Edinburgh, Scotland, October 2000.

* Indicates the major publication for the study 


\title{
CHARACTERISTICS OF STUDIES
}

\author{
Characteristics of included studies [ordered by study ID]
}

\author{
Agre 2003b
}

Methods

Aim of study:

To measure the effectiveness of three consent tools - videotape, computer and booklet formats- against the standard written consent form; and to compare different methods of delivering consent information across different phases of trials and in different patient populations

Study design:

Randomised controlled trial with four arms: videotape, booklet, computer instruction (CD) and standard information provision (control). (Note: data from booklet intervention arm not included in this review)

Methods of recruitment of participants:

Recruited during consent discussion for trials.

Inclusion/exclusion criteria for participation:

Inclusion criteria: Able to speak and read English; 18 years or older.

Exclusion criteria: None specified.

Ethics approval: Yes; participants signed a standard consent form approved by the study site's Institutional Review Board

Funding: Not specified.

Time span of trial: January 1999 to November 2001.

Statistical Methods:

Primary analysis: multiple regression to examine differences in knowledge across consent tools.

Secondary analyses: testing of multiplicative interaction effects (for differences in participant status, protocol complexity, trial phase, demographics, patient quality of life and family participation); hierarchical regression analysis; cluster analysis and 2-way ANOVA

Consumer involvement: No.

Participants

Description: Patients considering participation in 18 existing oncology trials; family and friends accompanying patients; and non-patient surrogates recruited from hospital waiting room. Only patients considering trial participation were included in this review.*

Geographic location: United States.

Setting: Hospital outpatient department (for consent discussion for trial participation)

Number of participants

Pretrial calculation of sample size:

Yes; authors considered power and required sample sizes in detail.

Authors stated that the level of power of the study was such that analyses would be sensitive to small differences in statistical tests and would allow exploration of findings across subgroups of patients and different protocols. Further information on sample size calculations provided to review authors and available on request

Eligible (total number approached to participate): Unclear.

Number excluded: 0. Authors note that all patients who were approached to participate were eligible for inclusion since the study was added on to existing clinical research studies

Number agreeing to participate/number refusing to take part: 155 patients agreed to participate. Number of people refusing to participate was not recorded but authors note that the number of refusals was low Number randomised to intervention: $106^{*}$

Video intervention: 53

Computer intervention (CD): 53

Number randomised to control: 49

Audio-visual presentation of information for informed consent for participation in clinical trials (Review) 
Number excluded post-randomisation: 0

Total: $155^{*}$

Number withdrawn: 0

Number lost to follow-up: 0

Number died: 0

Number included in final analysis: 155

Number included for each outcome:

Knowledge: 155 [49 control, 53 video, 53 computer]

Adequacy of follow-up: $100 \%$ for primary outcome [knowledge]

Age: average 59.5 years.

Gender: 74\% male (many prostate cancer patients), 26\% female

Ethnicity: Ethnicity of patient participants alone was not stated:12\% of total sample $(n=441)$ was of ethnic minority status, predominantly African American and Latino

Principal health problem or diagnosis: Patients - cancer. Details of type of cancer or stage of disease not given, although patients were enrolled in clinical oncology trials (therapeutic and non-therapeutic) of different phases

Other health problem/s: Not stated.

Stage of problem/illness: Not stated.

Treatment received/receiving: Not detailed, trials patients were enrolled in were both therapeutic (phase I, II or III) (15/18 trials) and non-therapeutic (3/18 trials)

Other social/demographic details:

Education level was mixed but generally high.

Educational range was assessed on $1-9$ scale $[1=<8$ th grade; $2=8$ th grade graduate; $3=$ some high school; 4 = high school graduate; 5 = business or technical college; $6=$ some college; $7=$ college degree; $8=$ some graduate school; 9 = professional degree $]$.

Patients: mean score 6.51.

Among patients, $45 \%$ had a family member(s) present who also participated in the informed consent process

Interventions

Aim of intervention:

To improve people's knowledge and understanding of trials to which they are considering consenting to participate

Format(s):

Video was non-interactive.

Structure of informational video and computer CD:

Length: Length of video was dependent on the oncology trial: the shortest was 10.24 minutes, the longest 21.39 minutes.

Language: English.

Content: Included description of the purpose of the research and research procedures; side-effects, risks, benefits and alternatives; confidentiality/ privacy; and the right to refuse/ withdraw from the study Number of times viewed: Once. Authors note that none of the participants viewed the video or CD more than once, although a small number of patients reviewed sections

Quality of video: Videos and CDs (computer intervention) were professionally produced; the videos used professional talent (no further details available)

Setting: Interventions were delivered in private consultation rooms

Target audience: Patients and/or their family members/ friends considering enrolling in oncology trials. Unrelated non-patient subjects waiting for patients in Surgical Day Hospital waiting area

Recipient: Consumers and family members; unrelated participants

Details of usual care: The standard consent document for each of the 18 oncology protocols was assessed 
for reading level, and amended if necessary to fall at the 8th grade reading level

Details of co-interventions: None, all patients signed a standard consent form after recruitment

Delivery of the intervention: No details provided.

Details of providers: All interventions were delivered by the principal investigator, or by one of two trained study research assistants

Intervention quality: Video and CD (computer intervention) were professionally produced. Note also that video and computer based interventions may improve the standardisation of information and presentation across study participants

Fidelity/integrity: Yes; all interventions were always delivered as intended

\begin{tabular}{|c|c|}
\hline Outcomes & $\begin{array}{l}\text { Primary outcomes: } \\
\text { Knowledge/ understanding. } \\
\text { Methods for follow-up of non-respondents: Not stated. } \\
\text { Authors' conclusions: } \\
\text { "Media consent tools are not a panacea for improving informed consent knowledge. Moreoever these } \\
\text { tools cannot simply be a conversion of standard consent information to another format...An important } \\
\text { finding... is that on average, no one in any group or demographic correctly answered more than two-thirds } \\
\text { of the knowledge questions.. There is great potential for using the computer as an interactive learning } \\
\text { tool that could engage patients and accommodate different styles of learning and different preferences for } \\
\text { information." }\end{array}$ \\
\hline Notes & $\begin{array}{l}\text { Limitations: } \\
\text { Knowledge test construction for specific oncology protocols/ trials may have yielded test of uneven diffi- } \\
\text { culty for different trial protocols. } \\
\text { Authors note that this study is limited to consideration of the informed consent process for traditional } \\
\text { medical/ oncological trials. Patients presented with genetic testing options or psychosocial protocols; those } \\
\text { with different medical or surgical issues; and those from ethnic minorities may all understand consent } \\
\text { information differently and the results of this study may therefore not be generalisable to these different } \\
\text { patient populations and/ or settings }\end{array}$ \\
\hline
\end{tabular}

Risk of bias

\begin{tabular}{|c|c|c|}
\hline Item & Authors' judgement & Description \\
\hline Allocation concealment? & No & D - Not used \\
\hline
\end{tabular}


To determine if research participants' understanding can be improved. Specifically, this study aimed to determine whether, and to what extent, innovative disclosure techniques can improve the amount and quality of information delivered to participants, and whether participants' understanding of the research can be improved

Study design:

Quasi-randomised controlled trial with four arms: unassisted disclosure (routine informed consent)(control); unassisted disclosure plus videotape (routine informed consent + instructional video); assisted disclosure and 'improved' videotape (second instructional video + additional information deemed appropriate to disclose by psychiatric investigator); neutral educator (note: data on this final study arm not included in the review)

Methods of recruitment of participants:

Potential participants for the current trial were participants in four existing psychiatric research studies (on depression, schizophrenia, social skills and borderline personality disorder). People were recruited as they were identified as prospective research participants by psychiatric investigators.

Inclusion/exclusion criteria for participation:

Included: participants in one of four psychiatric research studies.

Excluded: none stated.

Ethics approval:

Approval by the Institutional Review Boards at Western Psychiatric Institute and Clinic, University of Pittsburgh, and the University of California, Los Angeles

Funding:

Supported by the Foundations Fund for Research in Psychiatry

Time span of trial:

Approximately 1981 to 1982 . The four component psychiatric research studies ran for variable time periods (eg 6 weeks, 10 weeks) but data not provided for all studies

Statistical Methods:

Chi-square and one-way ANOVA with Tukey's post hoc testing were used to statistically assess differences between disclosure methods groups.

Ordinary least-squares multiple regression models were used for multivariate analysis to examine explanatory variables

Consumer involvement:

No.

Participants

Description: Psychiatric patients enrolled in psychiatric research studies

Geographic location: Pittsburgh, PA and Los Angeles, CA, USA

Setting: Two of four studies were conducted at a major university medical centre; the remaining two were conducted at a government psychiatric facility

Number of participants

Pretrial calculation of sample size: Unclear; authors state that the initial research design required 24 disclosure sessions per study (ie. with each disclosure method group containing six subjects from each of the four psychiatric studies). Delays in recruitment for the social skills and biploar disorder studies meant that numbers were decreased to 20 in each of these two studies

Eligible (total number approached to participate): Unclear.

Number excluded: Unclear. Note that author reply indicated that they did not recall any participants being excluded due to ineligibility, but that they could not be certain due to the length of time elapsed since the trial was conducted

Number agreeing to participate/number refusing to take part: 88 agreed to participate. Twelve subjects refused participation: one subject from each of the depression and schizophrenia studies; seven subjects from the social skills study; and three from the bipolar disorder study 
Number randomised to intervention ${ }^{* *}: 44$ ( $\mathrm{n}=22$ to the unassisted disclosure + videotape group; $\mathrm{n}=22$ to the assisted disclosure and improved videotape group)

Number randomised to control: 22

Number excluded post-randomisation: 0

Total: $66^{* *}$

Number withdrawn: 0

Number lost to follow-up: 0

Number died: 0

Number included in final analysis: $66^{* *}$

Number included for each outcome: $66^{* *}$

Adequacy of follow-up: $100 \%$ for each outcome

Age: mean 40.89 years (SD 16.43, range 18 to 76 years)

Gender: $70.5 \%$ male; $29.5 \%$ female.

Ethnicity: white $69.9 \%$; other $30.1 \%$

Principal health problem or diagnosis: Overall study population: schizophrenia 50\%, depression 27.3\%, borderline personality disorder $22.7 \%$

Other health problem/s: None stated.

Stage of problem/illness: Variable, as participants of four separate psychiatric research studies were included.

Depression study $(n=24)$ : participants were elderly people with major depression, randomly assigned to receive treatment with one of two antidepressant drugs for 10 weeks.

Schizophrenia study $(\mathrm{n}=24)$ : participants were community-based people with chronic schizophrenia, randomly assigned to treatment with a low or moderate dose of antipsychotic drug for several years.

Social skills training study $(\mathrm{n}=20)$ : participants were chronic schizophrenic outpatients, randomly assigned to control (normal day hospital) or to one of two experimental social skills training groups.

Borderline personality disorder study $(\mathrm{n}=20)$ : participants had a diagnosis of borderline personality disorder and were randomly assigned to treatment with placebo or one of two different psychotropic drugs for six weeks

Treatment received/receiving: See above.

Other social/demographic details: Education in years: mean 11.9 years (SD 2.16, range $=6$ to 18 years)

Interventions

Aim of intervention: To improve the amount and quality of the information given to prospective participants in psychiatric research trials, and to improve their understanding of research

Format(s): Videotape - 'standard' and 'improved' versions implemented, both formats non-interactive Structure of informational video: The videotapes employed were described as 'instructional' (standard) and 'improved' for the two different intervention components

Length: Unclear.

Language: English.

Content: Both 'instructional' (standard) and 'improved' video interventions included description of the study; the improved video incorporated feedback from research team on study description

Number of times viewed: Unclear.

Quality of video: Video quality was assessed as adequate by the research team. The videos were not professionally produced

Setting: Unclear.

Target audience: Potential psychiatric research participants

Recipient: Potential psychiatric research participants.

Details of usual care: Routine disclosure by psychiatric researcher; disclosures were independently rated for adequacy using a structured format 


\begin{tabular}{|c|c|c|}
\hline & \multicolumn{2}{|c|}{$\begin{array}{l}\text { Details of co-interventions: Routine disclosure process was delivered to all participants, including video } \\
\text { intervention groups } \\
\text { Delivery of the intervention: Unclear. } \\
\text { Details of providers: Investigator (psychiatric researcher); no further details } \\
\text { Intervention quality: Unclear. Video quality was assessed as adequate by the research team } \\
\text { Fidelity/integrity: The study design was such that interventions were intended to reflect what the re- } \\
\text { searchers involved in the four psychiatric trials wished to communicate to potential trial participants. The } \\
\text { differences between the two video intervention groups was solely whether feedback was provided to the } \\
\text { researchers presenting information to participants or not }\end{array}$} \\
\hline Outcomes & \multicolumn{2}{|c|}{$\begin{array}{l}\text { Primary outcomes: } \\
\text { Quality of research information disclosed. } \\
\text { Subject understanding of research. } \\
\text { Methods for follow-up of non-respondents: Unclear. } \\
\text { Authors' conclusions: } \\
\text { "Findings indicate that the use of experimental techniques generally increases the quality of information } \\
\text { delivered to prospective subjects...Subject understanding was also found to be significantly associated with } \\
\text { the quality of information provided... Innovative methods of information delivery...were generally supe- } \\
\text { rior to unassisted investigator disclosures in transmitting high-quality research information to prospective } \\
\text { subjects. However, our research also indicates that the impact of increased information on subject under- } \\
\text { standing may vary substantially across studies using different patient populations." }\end{array}$} \\
\hline Notes & \multicolumn{2}{|c|}{$\begin{array}{l}\text { Limitations: } \\
\text { Very limited information about the intervention characteristics provided in published report. Non-random } \\
\text { participant allocation may represent a source of bias although authors did examine groups for baseline } \\
\text { differences in demographic and illness characteristics and reported no differences across groups. Limited } \\
\text { information regarding blinding and other aspects of methods makes quality of study difficult to evaluate } \\
\text { Small study numbers in each group may mean insuffiicent power to detect an effect of the intervention } \\
\text { if one exists }\end{array}$} \\
\hline \multicolumn{3}{|l|}{ Risk of bias } \\
\hline Item & Authors' judgement & Description \\
\hline Allocation concealment? & No & D - Not used \\
\hline
\end{tabular}


Aim of study:

To study the effectiveness of videotape instruction in promoting the understanding of study conduct to potential study participants

Study design:

Randomised trial with two arms; group 1 (consent form + speak with study nurse + videotape) versus group 2 (consent form + speak with study nurse)

Methods of recruitment of participants:

Unclear: dates (1 May 1988 to 30 June 1989) refer to study duration, not specifically to the recruitment period. Unclear with respect to source of potential participants and to the process of invitation to participate Inclusion/exclusion criteria for participation in study:

Not stated explicitly; potential participants were individuals invited to participate in study of duodenal ulcer medication (short duration study, up to 8 weeks in duration)

Ethics approval: Unclear.

Funding: Not stated.

Time span of trial: 1 May 1988 to 30 June 1989.

Statistical Methods:

No formal statistical analysis of data. Percentages of participants in each group who answered all 10 questions correctly, 1 to 2 incorrect, 3 to 6 incorrect, 7 to 9 incorrect or all incorrect were tabulated as percentages

Consumer involvement: None stated.

Description:

Persons invited to participated in study of duodenal ulcer monitoring in response to study medication designed to promote ulcer healing

Geographic location: Unclear, although likely to be the USA.

Setting: Unclear.

Number of participants

Pretrial calculation of sample size: No.

Eligible (total number approached to participate): Unclear.

Number excluded: Unclear.

Number agreeing to participate/number refusing to take part: 200 (number refusing unclear).***

Number randomised to intervention: $100^{* * *}$

Number randomised to control: $100^{* * *}$

Number excluded post-randomisation: Unclear.

Total: Unclear.

Number withdrawn: Unclear.

Number lost to follow-up: Unclear.

Number died: Unclear.

Number included in final analysis: $200^{* * *}$

Number included for each outcome: $200^{* * *}$

Adequacy of follow-up: Appears to be $100 \%$, although numbers not directly reported

Age: Not reported.

Gender: Not reported.

Ethnicity: Not reported.

Principal health problem or diagnosis: Not reported directly but participants were potential participants for duodenal ulcer study

Other health problem/s: Not reported.

Stage of problem/illness: Not reported.

Treatment received/receiving: Duodenal ulcer drug - Sucralfate (Carafate)

Other social/demographic details: Not reported. 
Norris 1990 (Continued)

\begin{tabular}{|c|c|}
\hline Interventions & $\begin{array}{l}\text { Aim of intervention: } \\
\text { To promote knowledge and understanding of study conduct to potential study participants in the process } \\
\text { of obtaining informed consent } \\
\text { Format(s): Videotape; unclear whether interactive or non-interactive } \\
\text { Structure of informational video: } \\
\text { Length: Unclear. } \\
\text { Language: Unclear. } \\
\text { Content: } \\
\text { Included information on the study protocol and adherence to the study protocol } \\
\text { Number of times viewed: Unclear. } \\
\text { Quality of video: Unclear. } \\
\text { Setting: Video viewed as part of a group. } \\
\text { Target audience: Potential study participants. } \\
\text { Recipient: Potential study participants. } \\
\text { Details of usual care: Standard information provision and questions answered by study nurse } \\
\text { Details of co-interventions: Both groups studied the consent form and were able to ask questions of the } \\
\text { study nurse } \\
\text { Delivery of the intervention: } \\
\text { Video group (group 1): Participants studied the consent form and discussed it with the study nurse. They } \\
\text { then viewed the video, before consent being sought (ie prior to signing the consent form). Questionnaire } \\
\text { was then completed. } \\
\text { Control group (group 2): Participants studied the consent form and discussed it with the study nurse. } \\
\text { Consent was then sought and questionnaire completed } \\
\text { Details of providers: } \\
\text { Research nurse; no further detailed provided. } \\
\text { Intervention quality: Not reported. Although note that video intervention may improve the standardisation } \\
\text { of information and presentation across study groups } \\
\text { Fidelity/integrity: Not reported. }\end{array}$ \\
\hline
\end{tabular}

Outcomes

Primary outcomes:

Patient consent quiz regarding information in study patient information sheet (questionnaire)

Methods for follow-up of non-respondents:

Not reported.

Authors' conclusions:

"Utilization of audiovisual techniques, specifically videotape instruction, has been shown to increase a participant's understanding of the study and his/her role in it. While instructive videotapes can never supplement the role of the investigator or study nurse in communicating with the study participant, they can be extremely useful tools in making study participants 'truly informed,' thereby giving literal meaning to the term 'informed consent'."
Notes
Limitations:
Very limited outcomes assessed, with no mention of validity or reliability of outcome measures.
No description of the study population, or numbers followed through the study.
Very limited information reported regarding video content, design, delivery or evaluation

Risk of bias

\begin{tabular}{l|l} 
Item Authors' judgement $\quad$ Description & A
\end{tabular}

Audio-visual presentation of information for informed consent for participation in clinical trials (Review) 
Norris 1990 (Continued)

$\begin{array}{lll}\text { Allocation concealment? Unclear } & \text { B - Unclear }\end{array}$

Weston 1997

Methods

Aims of study:

(1) To evaluate the effect of the video on: (1) women's willingness to participate; (2) their views that the Term PROM Study was worthwhile; and (3) their ability to understand and retain information about: (a) prelabour rupture of the membranes at term; (b) the risks and benefits associated with the options for their care; and (c) various aspects of the study protocol.

(2) To evaluate the effect of a patient information video during the informed consent process of a perinatal trial

Study design:

Randomised trial with two arms; video group (written information + video) versus control group (written information only)

Methods of recruitment of participants:

Women were recruited from physicians' offices and clinics. Unclear of the process of invitation to participate

Inclusion/exclusion criteria for participation in study:

Inclusion criteria: English speaking; between 19 to 33 weeks' gestation; informed written consent.

Exclusion criteria: Women who had previously watched the Term PROM video. Note that the population for study was purposely chosen from gestational age groups that were not eligible for the TermPROM study so that recruitment would not interfere with or be confounded by the recruitment efforts of the TermPROM study

Ethics approval: Yes; approved by the Research Ethics Board, Women’s College Hospital Toronto, Ontario, Canada

Funding: Medical Research Council of Canada (MRC) grant \# MA-11392

Time span of trial: 28 June to 22 December 1994. Note that the study enrolled women during this period; actual time span of the trial not specified

Statistical Methods:

Contingency table chi-square analysis for categorical and binary variables;

Wilcoxon rank sum tests for group comparisons of continuous data that were not normally distributed. Two-sided p-values reported for all significance tests, with a value of 0.05 considered to indicate statistical significance.

Descriptive analysis of individual questionnaire outcomes/ items

Consumer involvement: Actual patients were involved in the production of the video intervention

Participants

Description:

Women potentially eligible to participate in the Term PROM study (the term prelabour rupture of membranes study) - a multicentre RCT comparing management policies of induction of labour versus expectant management for pregnant women with prelabour rupture of membranes at term, in terms of serious fetal/neonatal infection, caesarean section and patient satisfaction with care (ie pregnant women considering enrolling in perinatal trial)

Geographic location: Women's College Hospital, Toronto, Ontario, Canada

Setting: Most women watched video following baseline assessment and completed questionnaire 1 on the day of enrolment (in doctor's office) (83.3\% of video group; $95.8 \%$ of control group).

The remainder of women watched the video at home and completed questionnaire 1 at home

Number of participants

Pretrial calculation of sample size: Yes; to find an increase in willingness to participate (primary outcome)

Audio-visual presentation of information for informed consent for participation in clinical trials (Review) 
of $30 \%$, a sample size of $\mathrm{n}=45$ was required for each group (alpha $=0.05$, 2-tailed beta $=0.2$ )

Eligible (total number approached to participate): Data was not collected

Number excluded: Data was not collected.

Number agreeing to participate/number refusing to take part: 90 participated; data on number refusing participation was not collected

Number randomised to intervention: 42

Number randomised to control: 48

Number excluded post-randomisation: 0

Total: 90

Number withdrawn: 0

Number lost to follow-up: 5 (1 in video group [non-responder]; 4 in control group [2 preterm births and 2 non-responders])

Number died: 0

Number included in final analysis: 90 women for questionnaire 1, 85 women for questionnaire 2

Number included for each outcome:

Video group: $\mathrm{n}=42$ at baseline, $\mathrm{n}=41$ for follow-up measures

Control group: $\mathrm{n}=48$ at baseline, $\mathrm{n}=44$ for followup measures

Adequacy of follow-up: 100\% (90/90) for baseline measure 1; 94\% (85/90) for follow-up measure 2

Age:

Video group: median 31.4 years (range 21.8 to 39.5 )

Control group: median 31.8 years (range 18.0 to 31.3 )

Gender: All female.

Ethnicity: Not reported.

Principal health problem or diagnosis:

All pregnant women of gestational age range such that would not interfere with actual Term PROM study.

All 19 to 33 weeks gestation at enrolment

Other health problem/s: None stated.

Stage of problem/illness: None stated.

Treatment received/receiving: None stated.

Other social/demographic details

Schooling:

Video group: $\mathrm{n}=2(4.8 \%)$ high school or lower; $\mathrm{n}=40(95.2 \%)$ college or higher

Control group: $\mathrm{n}=4(8.3 \%)$ high school or lower; $\mathrm{n}=44(91.7 \%)$ college or higher

Parity:

Video group: $\mathrm{n}=23(54.8 \%)$ nulliparous; $\mathrm{n}=19(45.2 \%)$ one or more

Control group: $\mathrm{n}=26$ (54.2\%) nulliparous; $\mathrm{n}=22$ (45.8\%) one or more

Gestational age:

Video group: median 25 weeks (range 20.3 to 33.0 )

Control group: median 27.3 weeks (range 19.6 to 32.9 )

Interventions

Aim of intervention:

(1) To describe the objectives of the Term PROM study and to outline the care provided to participants in the different randomised groups.

(2) To increase willingness to participate in, feelings of worth of and knowledge of a perinatal trial Format(s): Videotape; non-interactive.

Structure of informational video:

Length: 10 minutes.

Language: English (Note not stated directly but assume English as study inclusion criteria specifies participants English speaking) 
Content: Included description of prelabour rupture of membranes at term; description of the study; personal account of trial participant; and invitation to participate in trial

Number of times viewed: Once.

Quality of video: Professionally produced, 'very high quality'

Setting: Doctor's office or at home. Most women watched video following baseline assessment and completed questionnaire 1 on the day of enrolment (in doctor's office) (83.3\% of video group; $95.8 \%$ of control group).

Some watched the video at home and completed questionnaire 1 at home

Target audience: Potential study participants.

Recipient: Pregnant women, potential study participants.

Details of co-interventions: Both groups received written information and were able to ask the study nurse questions. All consenting women were given the written information used during the consent process for the TermPROM study

Delivery of the intervention:

Video group: Video intervention delivered following routine information and discussion with study nurse. Baseline information (demographics etc) were collected; women were then given the written information sheet and questions were answered by the study nurse. Women then watched the video (duration 10 minutes).

Control group: Baseline information (demographics etc) was collected; women were then given the written information sheet and questions were answered by the study nurse

Details of providers:

The information contained in the video was presented by patients, families, nurses, the study's principal investigator and study staff, with narration by the professional narrator. Personal characteristics unclear. Study nurse was also involved in delivering information; no further details provided Intervention quality: Not reported. Although note that video intervention may improve the standardisation of information and presentation across study participants

Fidelity/integrity: Yes; the research assistant was present when participants viewed the video. All mothers stayed to watch the video

Outcomes

Primary outcome:

Willingness for future participation in the Term PROM study should they become eligible

Secondary outcomes:

Women's views on the importance of the Term PROM study

Knowledge of the study protocol

Knowledge of term prelabour rupture of membranes

Methods of follow-up for non-respondents:

After assessment 1, arrangements were made by the study nurse for each woman to complete the questionnaire again in 2 to 4 weeks. Follow-up for non-respondents unclear

Authors' conclusions:

"The showing of a patient information video may increase the willingness of women to participate in a research trial, and may have the additional effect of educating them about the health problem and specifics of the trial. This willingness to participate seems to decrease over time and therefore a patient information video may be most helpful at the time informed consent is requested."

Notes

Note study was duplicate publication: same study included in Weston (1995) abstract. Author reply (J. Weston, first author) confirmed the studies were the same

Limitations:

Sample did not include women with the specific health condition (Term PROM).

The effects of the video in women of different cultural or educational backgrounds is not clear. The sample

Audio-visual presentation of information for informed consent for participation in clinical trials (Review) 
studied included a high proportion of highly educated women, and authors note that the results may therefore not be generalisable beyond the current sample for these reasons

\section{Risk of bias}

\begin{tabular}{l|ll}
\hline Item & Authors' judgement & Description \\
\hline Allocation concealment? & Yes & A - Adequate \\
\hline
\end{tabular}

* Note that the study included a total of 441 participants. For this review, only data on patients was included. Data on non-patient surrogates $[\mathrm{n}=128]$ was excluded as they were not potential trial participants and therefore for them the trial was hypothetical. Data from family member/ friends accompanying patients to the informed consent discussion [ $\mathrm{n}=109]$ was excluded on the grounds that they were not expressly present to provide informed consent on the patient's behalf. A total of 204 patients was recruited to the trial. Data from the booklet intervention arm were also excluded from this review $[\mathrm{n}=49$ patients]: therefore in total data from 155 patients were included in this review.

** Note that study includes a total of 88 participants, including the neutral educator arm $[\mathrm{n}=22]$ not included in this review.

*** Note that the trial reports inconsistent participant numbers: stating initially that 278 participants signed consent forms and were randomised; and later, that 200 participants were randomised to two groups. We have used the number 200 participants, assuming 100 were allocated to each of two groups, for this review.

\section{Characteristics of excluded studies [ordered by study ID]}

\begin{tabular}{ll}
\hline Study & Reason for exclusion \\
\hline Agre 1994 & Intervention used for informed consent for therapeutic intervention rather than clinical research \\
\hline Agre 2002 & Not primary study; overview of research on informed consent \\
\hline Agre 2003a & Not primary study; overview of research in informed consent \\
\hline Barbour 1978 & Not randomised/ quasi-randomised (no comparison group) \\
\hline Benitez 2002 & No audio-visual intervention \\
\hline Benson 1985 & No audio-visual intervention (recording interaction only) \\
\hline Brandon 1991 & Not primary study, editorial \\
\hline Brown 2004 & Not randomised/ quasi-randomised; qualitative study \\
\hline Campbell 2004 & Hypothetical clinical trial participation \\
\hline Curbow 2004 & Not randomised/ quasi-randomised \\
\hline
\end{tabular}


(Continued)

\begin{tabular}{|c|c|}
\hline Dunbar 1989 & Not randomised/ quasi-randomised \\
\hline Dunn 2001 & Intervention did not include materials in audio-visual format \\
\hline Fisher 1991 & No audio-visual intervention; survey only \\
\hline Flory 2004 & Not randomised/ quasi-randomised; systematic review \\
\hline Fureman 1997 & Hypothetical clinical trial participation \\
\hline Hall 2001 & Not randomised/ quasi-randomised; qualitative study \\
\hline Harzstark 2001 & Intervention not associated with process of obtaining informed consent for research \\
\hline Hopper 1994 & Intervention used for informed consent for therapeutic intervention rather than clinical research \\
\hline Hougham 2003 & Not primary study; overview of research on informed consent \\
\hline Jefford 2002 & Not primary study; review \\
\hline Jensen 1993 & No audio-visual intervention \\
\hline Jepson 2001 & Not primary study; review \\
\hline Jimison 1998 & No audio-visual intervention evaluation, interactive tool development \\
\hline Joseph 2006 & Not randomised/ quasi-randomised (no comparison group) \\
\hline Koh 2001 & Not primary study; review \\
\hline Krogh 1999 & No audio-visual intervention; qualitative study \\
\hline Krouse 2001 & Not primary study; review \\
\hline Lenert 2000 & Does not assess informed consent for clinical trial participation \\
\hline Llewellyn 1995 & Hypothetical clinical trial participation \\
\hline Maslin 1998 & Intervention used for informed consent for therapeutic decision making rather than clinical research \\
\hline McPherson 2001 & Not primary study; systematic review \\
\hline McPherson 2002 & Not randomised/quasi-randomised; qualitative study \\
\hline McTiernan 1995 & No audio-visual intervention evaluation, description of informed consent process \\
\hline Mittal 2007 & Hypothetical clinical trial participation \\
\hline
\end{tabular}


(Continued)

\begin{tabular}{|c|c|}
\hline Morrow 1978 & No audio-visual intervention \\
\hline Moseley 2006 & Hypothetical clinical trial participation \\
\hline Palladino 2002 & Not primary study; review \\
\hline Pignone 2000 & Intervention used for informed consent for therapeutic intervention (screening) rather than clinical research \\
\hline Pletsch 2001a & Not randomised/ quasi-randomised; qualitative study \\
\hline Pletsch 2001b & Not randomised/ quasi-randomised; qualitative study \\
\hline Pletsch 2001c & Not randomised/ quasi-randomised; qualitative study \\
\hline Rangel 2002 & Not primary study; description of protocol \\
\hline Ruckdeschel 1996 & No audio-visual intervention \\
\hline Sachs 2003 & Not primary study; overview of research \\
\hline Sheldon 1993 & No audio-visual intervention \\
\hline Shurnas 2003 & No audio-visual intervention \\
\hline Simes 1986 & No audio-visual intervention \\
\hline Sugarman 1998 & Not primary study; review \\
\hline Thomas 2000 & Intervention used for informed consent for therapeutic intervention rather than clinical research \\
\hline Thornton 1993 & Not primary study; commentary on research meeting \\
\hline Tindall 1994 & No audio-visual intervention \\
\hline Tymchuk 1986 & Intervention not associated with process of obtaining informed consent for research \\
\hline Varnhagen 2005 & Hypothetical clinical trial participation \\
\hline Ventura 2003 & No audio-visual intervention \\
\hline Westreich 1995 & Intervention used for informed consent for therapeutic intervention rather than clinical research \\
\hline Wirshing 2005 & $\begin{array}{l}\text { Compares two forms of video presentation, not audio-visual intervention versus another form of information } \\
\text { provision }\end{array}$ \\
\hline Wragg 2000 & Hypothetical clinical trial participation \\
\hline
\end{tabular}


DATA ANDANALYSES

This review has no analyses.

\section{ADDITIONAL TABLES}

Table 1. Details of interventions

\begin{tabular}{ll} 
Study ID & Intervention \\
\hline Agre 2003b & $\begin{array}{l}\text { AIM } \\
\text { To improve people's knowledge and understanding of tri- } \\
\text { als to they are considering consenting to participate. }\end{array}$
\end{tabular}

THEORETICAL BASIS

Literature cited identifies lack of understanding of research trials by participants in clinical research.

\section{DEVELOPMENT}

The consent document for each of the 18 oncology protocols was assessed for reading level, and amended if necessary to fall at the 8th grade reading level. Language for the basic content in all consent tools was identical, with summary/ review sections added to the video and computer-assisted instructional program formats. The consent form was used as the basis for scripts developed by the researchers for a professionally-produced computerassisted instructional program and video. The videos used professional talent.

\section{CONTENT}

Content of the interventions was based on the trials for which consent was being sought. However, all had identical sections based on the standard informed consent procedure for clinical trials used at the institution (Memorial Sloan-Kettering Cancer Centre) (for example, the purpose of the research; description of the research procedures; side-effects, risks and benefits of treatment; compensation in the case of research-related injury; alternatives; financial costs; confidentiality/ privacy; the right to refuse or withdraw from the study; termination; conclusions)

\section{Control}

\section{USUAL CARE:}

The consent document for each of the 18 oncology protocols was assessed for reading level, and amended if necessary to fall at the 8 th grade reading level, which was deemed as the appropriate target population level for the study site

\section{USUAL CARE:}

To improve the amount and quality of information given to prospective participants in psychiatric research trials, and to improve their understanding of research.

Specifically, four different disclosure techniques were examined, each aiming to improve the amount and quality of information given to prospective participants: unas-
Routine information provided as psychiatric researcher's standard disclosure to trial participants 
sisted disclosure (control, routine informed consent procedure); unassisted disclosure plus videotape (routine informed consent plus instructional videotape); assisted disclosure plus 'improved' videotape (additional information psychatric investigator deemed appropriate to disclose plus improved videotape); and neutral educator (not considered in this review).

\section{THEORETICAL BASIS}

Literature cited identifies psychiatric patients as a population 'at risk' in relation to the informed consent process. The intervention was developed specifically to determine whether video augmentation of the informed consent process would improve participant understanding.

\section{DEVELOPMENT}

The intervention was developed in-house, by the research team.

\section{CONTENT}

The videotapes employed were described as 'instructional' (standard) and 'improved' for the two different intervention components. The standard video format involved the principal investigator or other designated project staff from the psychiatric trials describing the study as he/she chose to do so. This typically reflected the usual presentation made to subjects at the time of consent. The improved video format included feedback from the research team about areas of the disclosure that could be improved or requried greater emphasis. Following this feedback, the second 'improved' video format was produced

To promote knowledge and understanding of study conduct to potential study participants in the process of obtaining informed consent.

Standard information provision:

Both groups studied the consent form and had discussion with the study nurse

\section{THEORETICAL BASIS}

Not reported.

\section{DEVELOPMENT \\ Unclear.}

\section{CONTENT}

Included information on the following: compliance with dosing schedules; maintenance of diary cards; adherence to specific antacid limitations; presence at scheduled follow-up visits; procedures to be used; possible adverse reactions; study consent forms; and who to call for infor- 
mation

Weston 1995 AIM

(1) To describe the objectives of the Term PROM study and to outline the care provided to participants in the different randomised study groups.

(2) To increase willingness to participate in, feelings of worth of and knowledge of a perinatal trial.

\section{THEORETICAL BASIS:}

Prior literature cited deals with the importance of adequately informed consent.

\section{DEVELOPMENT}

The video was professionally produced by Edwin Medical Communications Inc., a video production house specialising in medical education videos.

Patient information video used patients and their families, nurses, the study's principal investigator and study staff. Narration was by a professional narrator; the video was produced commercially and of 'high quality'.

\section{CONTENT}

Description of the medical condition, prelabour rupture of membranes at term (Term PROM).

Description of the study, including: the manoeuvre showing actual patients receiving each treatment, the risks and benefits of all study groups, the benefits of participating in clinical research and important aspects of the trial protocol, described by the principal investigator.

Actual trial participant described why she had participated in the study, the contribution she felt it made to medical science and to future women. An invitation to participate in the study and instructions on where to obtain further information on study participation were also included
USUAL CARE:

Standard information provision:

Written information was provided: this was written to be understood by women with 8 th grade education, with questions answered by the study nurse

Table 2. Details of outcome measures

\section{STUDY ID OUTCOMES}

Agre 2003b OUTCOME: Knowledge/ understanding

METHOD: Proportion of correct responses on a 12-15 item multiple choice knowledge quiz (created for each of the 18 oncology protocols). Completed by participants following the informed consent intervention delivery. TIMING: Assessed immediately after the informed consent intervention.

VALIDATED: Authors state that face validity was informally assessed by the clinical research study principal investigator.

RELIABILITY ASSSESSED: Authors note that the outcome measure was a quiz on the content of the intervention, answers to which were verbatim in the text, such that no inference was needed and no separate reliability testing was performed. 
Table 2. Details of outcome measures (Continued)

ADVERSE EVENTS OR HARMS:

Not reported.

Benson 1988 OUTCOME: Subject understanding of research

METHOD: Subject interview; unclear who conducted interview. Subject understanding assessed based on 15 items, each rated on a 3 -point scale $(0=$ poor to 2 = fair $)$.

TIMING: Imediately following informed consent disclosure session, and at approximately 2 weeks after disclosure session. Note that only data from interviews immediately after the disclosure session were presented (no data at 2 week follow-up reported).

VALIDATED: No, no independent checks on validity.

RELIABILITY ASSSESSED: Yes; a structured rating system was used for each outcome and reliability demonstrated, alpha 0.81 .

OUTCOME: Quality of research information disclosed

METHOD: Completeness of communicated information items were each rated on a 4-point scale $(0=$ no information disclosed to 3 = information disclosed superior to the written consent form), with the study's written consent form serving as the standard reference point; rated by observer of disclosure session.

TIMING: During informed consent disclosure session.

VALIDATED: No independent checks on validity.

RELIABILITY ASSSESSED: Yes; a structured rating system was used for each outcome and reliability demonstrated, alpha 0.84

ADVERSE EVENTS OR HARMS:

Not reported.

Norris 1990 OUTCOME: Patient consent quiz (knowledge of information in study patient information sheet).

METHOD: Questionnaire, 10 items; self completed by participant

TIMING: Following completion of information provision, assessed immediately after signing the consent form (at completion of videotape for Group 1 [intervention] or after speaking with study nurse for Group 2 [control]).

VALIDATED: Unclear

RELIABILITY ASSESSED: Unclear

ADVERSE EVENTS OR HARMS:

Not reported.

Weston 1997 OUTCOME: Knowledge of study protocol

METHOD: Questionnaire, 5 items; self-completed by participant.

TIMING: Questionnaire 1 completed at baseline after viewing video; questionnaire 2 completed at 2 to 4 weeks following recruitment (video group: median 17 days, range 9 to 15 days; control group: median 16.5 days, range 10 to 56 days).

VALIDATED: No

RELIABILITY ASSESSED: No

OUTCOME: Knowledge of PROM

METHOD: Questionnaire, 6 items; self -completed by participant

TIMING: Questionnaire 1 completed at baseline after viewing video; questionnaire 2 completed at 2 to 4 weeks following recruitment (video group: median 17 days, range 9 to 15 days; control group: median 16.5 days, range 10 to 56 days). 
Table 2. Details of outcome measures (Continued)

\author{
VALIDATED: No. \\ RELIABILITY ASSESSED: No.
}

OUTCOME: Willingness for future participation.

METHOD: Questionnaire, 1 item; self-completed by participant

TIMING: Questionnaire 1 completed at baseline after viewing video; questionnaire 2 completed at 2 to 4 weeks following recruitment (video group: median 17 days, range 9 to 15 days; control group: median 16.5 days, range 10 to 56 days).

VALIDATED: No

RELIABILITY ASSESSED: No

OUTCOME: Importance of study.

METHOD: Questionnaire, 1 item; self-completed by participant

TIMING: Questionnaire 1 completed at baseline after viewing video; questionnaire 2 completed at 2 to 4 weeks following recruitment (video group: median 17 days, range 9 to 15 days; control group: median 16.5 days, range 10 to 56 days).

VALIDATED: No

RELIABILITY ASSESSED: No

Note: All outcomes assessed as separate items on the one questionnaire.

ADVERSE EVENTS OR HARMS:

Not reported.

Table 3. Quality of included studies

\begin{tabular}{|c|c|c|c|c|c|c|c|}
\hline STUDY ID & $\begin{array}{l}\text { RANDOMI- } \\
\text { SATION }\end{array}$ & $\begin{array}{l}\text { AL- } \\
\text { LOC. CON- } \\
\text { CEALMENT }\end{array}$ & BLINDING & $\begin{array}{l}\text { FOLLOW } \\
\text { UP }\end{array}$ & $\begin{array}{l}\text { BASELINE } \\
\text { COMP. }\end{array}$ & $\begin{array}{l}\text { OUTCOME } \\
\text { MEASURES }\end{array}$ & $\begin{array}{l}\text { ITT ANALY- } \\
\text { SIS }\end{array}$ \\
\hline Agre 2003b & $\begin{array}{l}\text { RANDOMI- } \\
\text { SATION } \\
\text { Clinical } \\
\text { research } \\
\text { database using } \\
\text { randomly per- } \\
\text { muted block } \\
\text { scheme. } \\
\text { METHOD } \\
\text { Computer- } \\
\text { generated ran- } \\
\text { domisation se- } \\
\text { quence. }\end{array}$ & $\begin{array}{l}\text { ALLOCA- } \\
\text { TION CON- } \\
\text { CEALMENT } \\
\text { No } \\
\text { METHOD } \\
\text { Not applicable }\end{array}$ & $\begin{array}{l}\text { BLINDING } \\
\text { PARTICI- } \\
\text { PANT: No } \\
\text { PROVIDER: } \\
\text { No } \\
\text { OUTCOME } \\
\text { ASSESSOR: } \\
\text { No }\end{array}$ & $\begin{array}{l}\text { FOLLOW } \\
\text { UP } \\
100 \% \text { follow- } \\
\text { up achieved. } \\
\text { Details: } \\
325 \text { partic- } \\
\text { ipants were al- } \\
\text { located to con- } \\
\text { trol group and } \\
\text { either one of } \\
\text { two interven- } \\
\text { tions } \\
\text { groups. Out- } \\
\text { comes were as- } \\
\text { sessed imme- } \\
\text { diately follow- }\end{array}$ & $\begin{array}{l}\text { BASELINE } \\
\text { COMPARA- } \\
\text { BILITY } \\
\text { Yes; interven- } \\
\text { tion groups } \\
\text { were as- } \\
\text { sessed as com- } \\
\text { parable on age, } \\
\text { sex and educa- } \\
\text { tional level }\end{array}$ & $\begin{array}{l}\text { VALIDITY } \\
\text { OF OUT- } \\
\text { COMEMEA- } \\
\text { SURES } \\
\text { Yes: } \\
\text { assessment } \\
\text { of face validity } \\
\text { was performed } \\
\text { informally by } \\
\text { principal } \\
\text { investigator. } \\
\text { RELI- } \\
\text { ABILITY OF } \\
\text { OUTCOME } \\
\text { MEASURES } \\
\text { No; not }\end{array}$ & $\begin{array}{l}\text { INTEN- } \\
\text { TION TO } \\
\text { TREAT } \\
\text { ANALYSIS } \\
\text { Yes }\end{array}$ \\
\hline
\end{tabular}

Audio-visual presentation of information for informed consent for participation in clinical trials (Review) 
Table 3. Quality of included studies (Continued)

$$
\begin{aligned}
& \text { ing delivery of } \\
& \text { the interven- } \\
& \text { tion during } \\
& \text { the } \\
& \text { informed con- } \\
& \text { sent session }
\end{aligned}
$$

assessed separately but authors note that as outcomes were assessed as verbatim knowledge responses from text, no interpretation of results was needed and re-

\begin{tabular}{|c|c|c|c|c|c|c|c|}
\hline Benson 1988 & $\begin{array}{l}\text { RANDOMI- } \\
\text { SATION } \\
\text { Not used. } \\
\text { METHOD } \\
\text { Quasi- } \\
\text { random } \\
\text { allocation: se- } \\
\text { quential allo- } \\
\text { cation to study } \\
\text { groups }\end{array}$ & $\begin{array}{l}\text { ALLOCA- } \\
\text { TION CON- } \\
\text { CEALMENT } \\
\text { Not used. } \\
\text { METHOD } \\
\text { Not } \\
\text { applicable. }\end{array}$ & $\begin{array}{l}\text { BLINDING } \\
\text { PARTICI- } \\
\text { PANT: No } \\
\text { PROVIDER: } \\
\text { No } \\
\text { OUTCOME } \\
\text { ASSESSOR: } \\
\text { No }\end{array}$ & $\begin{array}{l}\text { FOLLOW } \\
\text { UP } \\
100 \% \text { follow- } \\
\text { up achieved } \\
\text { for arms eligi- } \\
\text { ble for this re- } \\
\text { view } \\
\text { Details: } \\
88 \\
\text { ipants were al- } \\
\text { located to four } \\
\text { disclosure } \\
\text { groups. } \\
66 \text { were eligi- } \\
\text { ble for this re- } \\
\text { view. } \\
\text { Out- } \\
\text { comes were as- } \\
\text { sessed imme- } \\
\text { diately after } \\
\text { disclosure ses- } \\
\text { sions, } \\
\text { two weeks and } \\
\text { at two to six } \\
\text { months post- } \\
\text { disclosure ses- } \\
\text { sion; the cur- } \\
\text { rent study re- } \\
\text { ports on out- }\end{array}$ & $\begin{array}{l}\text { BASELINE } \\
\text { COMPARA- } \\
\text { BILITY } \\
\text { Yes; groups as- } \\
\text { sessed as com- } \\
\text { parable on age, } \\
\text { race, sex and } \\
\text { patient ed- } \\
\text { ucation/ social } \\
\text { class. Groups } \\
\text { also compara- } \\
\text { ble on baseline } \\
\text { psychopathol- } \\
\text { ogy scores as- } \\
\text { sessed using } \\
\text { the Brief Psy- } \\
\text { chatric Rating } \\
\text { Scale }\end{array}$ & $\begin{array}{l}\text { VALIDITY } \\
\text { OF OUT- } \\
\text { COME MEA- } \\
\text { SURES } \\
\text { Unclear } \\
\text { RELI- } \\
\text { ABILITY OF } \\
\text { OUTCOME } \\
\text { MEASURES } \\
\text { Yes; quality of } \\
\text { research infor- } \\
\text { mation relia- } \\
\text { bility rating al- } \\
\text { pha = 0.84; re- } \\
\text { liability of } \\
\text { subject under- } \\
\text { standing rat- } \\
\text { ing alpha = } 0 . \\
81\end{array}$ & $\begin{array}{l}\text { INTEN- } \\
\text { TION TO } \\
\text { TREAT } \\
\text { ANALYSIS } \\
\text { Yes } \\
\text { (based } \\
\text { on those arms } \\
\text { eligible for this } \\
\text { review) }\end{array}$ \\
\hline
\end{tabular}
li-

ability testing was therefore not performed separately 
Table 3. Quality of included studies (Continued)

\begin{tabular}{|c|c|c|c|c|c|c|c|}
\hline & & & & $\begin{array}{l}\text { comes assessed } \\
\text { immedi- } \\
\text { ately after and } \\
\text { two weeks af- } \\
\text { ter the disclo- } \\
\text { sure session }\end{array}$ & & & \\
\hline Norris 1990 & $\begin{array}{l}\text { RANDOMI- } \\
\text { SATION } \\
\text { Unclear } \\
\text { METHOD } \\
\text { Not specified }\end{array}$ & $\begin{array}{l}\text { ALLOCA- } \\
\text { TION CON- } \\
\text { CEALMENT } \\
\text { Unclear } \\
\text { METHOD } \\
\text { Not specified }\end{array}$ & $\begin{array}{l}\text { BLINDING } \\
\text { PARTIC- } \\
\text { IPANT: Un- } \\
\text { clear } \\
\text { PROVIDER: } \\
\text { Unclear } \\
\text { OUTCOME } \\
\text { ASSESSOR: } \\
\text { Unclear }\end{array}$ & $\begin{array}{l}\text { FOLLOW } \\
\text { UP } \\
\text { Apparently } \\
100 \% \\
\text { Note that par- } \\
\text { ticipants num- } \\
\text { bers were not } \\
\text { reported } \\
\text { explicitly. Au- } \\
\text { thors reported } \\
\text { that } 200 \text { par- } \\
\text { ticipants were } \\
\text { randomly as- } \\
\text { signed } \\
\text { to two groups; } \\
\text { this im- } \\
\text { plies } 100 \text { par- } \\
\text { ticipants } \\
\text { per group al- } \\
\text { though num- } \\
\text { bers are not re- } \\
\text { ported } \\
\text { directly. } \\
\text { Percentage } \\
\text { data for out- } \\
\text { comes assessed } \\
\text { reported only }\end{array}$ & $\begin{array}{l}\text { BASELINE } \\
\text { COMPARA- } \\
\text { BILITY } \\
\text { Un- } \\
\text { clear: No base- } \\
\text { line character- } \\
\text { istics reported. }\end{array}$ & $\begin{array}{l}\text { VALIDITY } \\
\text { OF OUT- } \\
\text { COME MEA- } \\
\text { SURES } \\
\text { Unclear } \\
\text { RELI- } \\
\text { ABILITY OF } \\
\text { OUTCOME } \\
\text { MEASURES } \\
\text { Unclear }\end{array}$ & $\begin{array}{l}\text { INTEN- } \\
\text { TION TO } \\
\text { TREAT } \\
\text { ANALYSIS } \\
\text { No } \\
\text { statistical anal- } \\
\text { ysis was per- } \\
\text { formed, per- } \\
\text { centage data } \\
\text { reported only }\end{array}$ \\
\hline Weston 1997 & $\begin{array}{l}\text { RANDOMI- } \\
\text { SATION } \\
\text { Yes } \\
\text { METHOD } \\
\text { Random } \\
\text { numbers table } \\
\text { held at central } \\
\text { Data Coordi- } \\
\text { nating Centre }\end{array}$ & $\begin{array}{l}\text { ALLOCA- } \\
\text { TION CON- } \\
\text { CEALMENT } \\
\text { Adequate } \\
\text { METHOD } \\
\text { Telephone, } \\
\text { centrally held } \\
\text { records. }\end{array}$ & $\begin{array}{l}\text { BLINDING } \\
\text { PARTICI- } \\
\text { PANT: No } \\
\text { PROVIDER: } \\
\text { No } \\
\text { OUTCOME } \\
\text { ASSESSOR: } \\
\text { No }\end{array}$ & $\begin{array}{l}\text { FOLLOW } \\
\text { UP } \\
90 / 90(100 \%) \\
\text { completed } \\
\text { baseline ques- } \\
\text { tionnaires } \\
85 / 90 \text { (94\%) } \\
\text { completed fol- } \\
\text { low-up ques- } \\
\text { tionnaires } \\
\text { (2 to } 4 \text { weeks } \\
\text { later) }\end{array}$ & $\begin{array}{l}\text { BASELINE } \\
\text { COMPARA- } \\
\text { BILITY } \\
\text { Yes; groups } \\
\text { compa- } \\
\text { rable on ma- } \\
\text { ternal age, me- } \\
\text { dian gestation, } \\
\text { parity, educa- } \\
\text { tion and pre- } \\
\text { vious knowl- } \\
\text { edge of study }\end{array}$ & $\begin{array}{l}\text { VALIDITY } \\
\text { OF OUT- } \\
\text { COME MEA- } \\
\text { SURES } \\
\text { No } \\
\text { RELI- } \\
\text { ABILITY OF } \\
\text { OUTCOME } \\
\text { MEASURES } \\
\text { No }\end{array}$ & $\begin{array}{l}\text { INTEN- } \\
\text { TION TO } \\
\text { TREAT } \\
\text { ANALYSIS } \\
\text { Yes }\end{array}$ \\
\hline
\end{tabular}




\section{A P P E N D I C ES}

\section{Appendix I. MEDLINE search strategy}

1. exp video recording/ or exp audiovisual aids/

2. (video $\$$ or audiovisual\$).tw.

3. (interactive multimedia or interactive media).tw.

4. or/1-3

5. informed consent/ or consent.tw.

6. exp truth disclosure/ or (truth adj disclosure).mp.

7. disclosure/ or disclos\$.tw.

8. exp communication/ or communicat $\$$.tw.

9. (inform $\$$ adj 1 trial\$).tw.

10. decision making/ or (decision or decid\$).tw.

11. or $/ 5-10$

12. exp clinical trials/ or (clinic $\$$ adj3 trial\$).tw.

13. ((vaccine $\$$ or $\operatorname{drug} \$)$ adj3 trial\$).tw.

14. therapeutic human experimentation/ or human experimentation/

15. patient selection/ or (select $\$$ adj3 patient $\$$ ).tw.

16. patient participation/ or (participat $\$$ adj3 patient $\$$ ).tw.

17. (participat $\$$ adj3 (consumer $\$$ or client $\$$ )).tw.

18. research subjects/ or (research $\$$ adj1 subject $\$$ ).tw.

19. researcher subject relations/

20. (research $\$$ or experiment $\$$ or investigat $\$$ ).tw.

21. or/ $12-20$

22. 4 and 11 and 21

23. randomized controlled trial.pt.

24. controlled clinical trial.pt.

25. randomized controlled trials.sh.

26. random allocation.sh.

27. double blind method.sh.

28. single blind method.sh.

29. or/23-28

30. animals/ not (human/ and animals/)

31. 29 not 30

32. clinical trial.pt.

33. exp clinical trials/

34. (clin\$ adj25 trial\$).ti,ab.

35. ((singl\$ or doubl\$ or trebl\$ or tripl\$) adj25 (blind $\$$ or mask $\$)$ ).ti,ab.

36. placebos.sh.

37. placebo\$.ti,ab.

38. random $\$ . t i, a b$.

39. research design.sh.

40. or/32-39

41.40 not 30

42.31 or 41

43. 22 and 42 


\section{Appendix 2. EMBASE search strategy}

1. videorecording/ or exp audiovisual equipment/

2. (video $\$$ or audiovisual $\$$ ).tw.

3. (interactive multimedia or interactive media).tw.

4. or/1-3

5. informed consent/ or consent.tw.

6. interpersonal communication/

7. disclos\$.tw.

8. communicat\$.tw.

9. decision making/ or (decision or decid\$).tw.

10. (inform\$ adj1 trial\$).tw.

11. or $/ 5-10$

12. exp clinical trial/ or (clinic $\$$ adj3 trial\$).tw.

13. ((vaccine $\$$ or drug $\$$ ) adj3 trial\$).tw.

14. human experiment/ or human experiment $\$$.tw.

15. patient selection/ or (select\$ adj3 patient\$).tw.

16. (patient $\$$ adj3 participat $\$$ ).tw.

17. ((consumer\$ or client\$) adj3 participat\$).tw.

18. (research $\$$ adj 1 subject $\$$ ).tw.

19. (research $\$$ or experiment $\$$ or investigat $\$$ ).tw.

20. or/12-19

21. 4 and 11 and 20

22. randomized controlled trial/

23. random $\$$.tw.

24. exp controlled study/

25. double blind procedure/

26. single blind procedure/

27. crossover procedure/

28. latin square design/

29. multicenter study/

30. ((clinical or controlled or comparative or placebo or prospective or random $\$$ ) adj3 (trial or study)).tw.

31. ((single $\$$ or doubl $\$$ or trebl\$ or tripl\$) adj7 (blind $\$$ or mask $\$)$ ).tw.

32. (crossover\$ or cross-over\$ or (cross adj1 over\$)).tw.

33. ( (allocat $\$$ or allot $\$$ or assign $\$$ or divid $\$$ ) adj3 (condition $\$$ or experiment $\$$ or intervention $\$$ or treatment $\$$ or therap $\$$ or control\$ or group $\$)$ ).tw.

34. or/22-33

35.21 and 34

\section{Appendix 3. CINAHL search strategy}

1 exp Videorecording/

2 exp Audiovisuals/

3 (video $\$$ or audiovisual\$).tw.

4 (interactive medi\$ or interactive multimedi\$).tw.

5 or/ $1-4$

6 exp "CONSENT (RESEARCH)"/ or exp CONSENT/

7 exp Truth Disclosure/

8 (truth adj disclosure).tw.

9 disclos $\$$.tw.

10 consent.tw.

11 exp COMMUNICATION/

12 communicat\$.tw.

Audio-visual presentation of information for informed consent for participation in clinical trials (Review)

Copyright $\odot 2009$ The Cochrane Collaboration. Published by John Wiley \& Sons, Ltd. 
13 (inform\$ adj1 trial\$).tw.

14 exp Decision Making/

15 decision.mp. or decid\$.tw. [mp=title, subject heading word, abstract, instrumentation]

16 or/6-15

17 exp Clinical Trials/

18 (clinic $\$$ adj3 trial\$).tw.

19 ((vaccine $\$$ or drug $\$$ ) adj3 trial\$).tw.

20 exp Clinical Research/

21 exp Patient Selection/

22 (select\$ adj3 patient\$).tw.

23 exp Consumer Participation/

24 (participat\$ adj3 consumer\$).tw.

25 (participat\$ adj3 (client\$ or patient\$)).tw.

26 exp Research Subjects/

27 (research\$ adj1 subject\$).tw.

28 (research\$ or experiment\$ or investigat\$).tw.

29 or/ $17-28$

305 and 16 and 29

31 randomi?ed controlled trial\$.af.

32 random $\$$.tw.

33 clinical trial.pt.

34 exp Clinical Trials/

35 (clin\$ adj25 trial\$).tw.

36 ((singl\$ or doubl\$ or treb\$ or tripl\$) adj25 (blind $\$$ or mask $\$)$ ).tw.

37 exp PLACEBOS/

38 placebo $\$ . t w$.

39 exp Study Design/

40 or/31-39

4130 and 40

\section{Appendix 4. PsycINFO 2004 search strategy}

1. educational audiovisual aids/

2. videotape instruction/

3. audiovisual instruction/

4. (video\$ or audiovisual\$).tw.

5. (interactive multimedia or interactive media).tw.

6. or/ $1-5$

7. informed consent/ or consent.tw.

8. duty to warn/

9. (disclosure or disclos $\$$ ).tw.

10. communicat\$.tw.

11. interpersonal communication/

12. decision making/ or (decision or decid\$).tw.

13. (inform\$ adj1 trial\$).tw.

14. or/7-13

15. (clinic\$ adj3 trial\$).tw.

16. (random $\$$ adj 3 trial $\$$ ).tw.

17. ((vaccine\$ or drug\$) adj3 trial\$).tw.

18. patient selection/ or (select $\$$ adj3 patient $\$$ ).tw.

19. client participation/ or (client $\$$ adj3 participat $\$$ ).tw.

20. ((patient\$ or consumer\$) adj3 participat\$).tw.

Audio-visual presentation of information for informed consent for participation in clinical trials (Review)

Copyright @ 2009 The Cochrane Collaboration. Published by John Wiley \& Sons, Ltd. 
21. experiment volunteers/

22. experimental subjects/

23. (research $\$$ adj 1 subject\$).tw.

24. (research $\$$ or experiment $\$$ or investigat $\$$ ).tw.

25. or/ $15-24$

26. 6 and 14 and 25

27. randomi?ed controlled trial\$.af.

28. random $\$$.tw.

29. (clin\$ adj25 trial\$).tw.

30. ((singl\$ or doubl\$ or treb\$ or tripl\$) adj25 (blind\$ or mask\$)).tw.

31. placebo/

32. placebo $\$ . t w$.

33. or $/ 27-32$

34.26 and 33

\section{Appendix 5. PsycINFO 2006 search strategy}

1. videorecording/ or exp audiovisual equipment/

2. (video $\$$ or audiovisual\$).tw.

3. (interactive multimedia or interactive media).tw.

4. or/1-3

5. informed consent/ or consent.tw.

6. interpersonal communication/

7. disclos\$.tw.

8. communicat\$.tw.

9. decision making/ or (decision or decid\$).tw.

10. (inform\$ adj1 trial\$).tw.

11. or $/ 5-10$

12. exp clinical trial/ or (clinic $\$$ adj3 trial\$).tw.

13. ((vaccine\$ or drug\$) adj3 trial\$).tw.

14. human experiment/ or human experiment\$.tw.

15. patient selection/ or (select $\$$ adj3 patient $\$$ ).tw.

16. (patient $\$$ adj3 participat $\$$ ).tw.

17. ((consumer\$ or client\$) adj3 participat\$).tw.

18. (research\$ adj1 subject\$).tw.

19. (research $\$$ or experiment $\$$ or investigat $\$$ ).tw.

20. or/12-19

21. 4 and 11 and 20

22. randomized controlled trial/

23. random\$.tw.

24. exp controlled study/

25. double blind procedure/

26. single blind procedure/

27. crossover procedure/

28. latin square design/

29. multicenter study/

30. ((clinical or controlled or comparative or placebo or prospective or random $\$$ ) adj3 (trial or study)).tw.

31. ((single $\$$ or doubl\$ or trebl\$ or tripl\$) adj7 (blind\$ or mask $\$)$ ).tw.

32. (crossover\$ or cross-over $\$$ or (cross adj1 over $\$)$ ).tw.

33. ( (allocat $\$$ or allot $\$$ or assign $\$$ or divid $\$$ ) adj3 (condition $\$$ or experiment $\$$ or intervention $\$$ or treatment $\$$ or therap\$ or control\$ or group\$)).tw.

34. or/22-33

Audio-visual presentation of information for informed consent for participation in clinical trials (Review)

Copyright $\odot 2009$ The Cochrane Collaboration. Published by John Wiley \& Sons, Ltd. 
35. 21 and 34

36. limit 35 to $y \mathrm{r}=$ "2004 - 2006"

37. videorecording/ or exp audiovisual equipment/

38. (video $\$$ or audiovisual $\$$ ).tw.

39. (interactive multimedia or interactive media).tw.

40. or/37-39

41. informed consent/ or consent.tw.

42. interpersonal communication/

43. disclos\$.tw.

44. communicat\$.tw.

45. decision making/ or (decision or decid\$).tw.

46. (inform\$ adj1 trial\$).tw.

47. or/41-46

48. exp clinical trial/ or (clinic $\$$ adj3 trial\$).tw.

49. ((vaccine $\$$ or drug $\$$ ) adj3 trial\$).tw.

50. human experiment/ or human experiment $\$$.tw.

51. patient selection/ or (select $\$$ adj3 patient $\$$ ).tw.

52. (patient $\$$ adj3 participat $\$$ ).tw.

53. ((consumer $\$$ or client $\$$ ) adj3 participat $\$$ ).tw.

54. (research $\$$ adj 1 subject $\$$ ).tw.

55. (research $\$$ or experiment $\$$ or investigat $\$$ ).tw.

56. or $/ 48-55$

57. 40 and 47 and 56

58. randomized controlled trial/

59. random\$.tw.

60. exp controlled study/

61. double blind procedure/

62. single blind procedure/

63. crossover procedure/

64. latin square design/

65. multicenter study/

66. ((clinical or controlled or comparative or placebo or prospective or random $\$$ ) adj3 (trial or study)).tw.

67. ((single $\$$ or doubl $\$$ or trebl\$ or tripl\$) adj7 (blind $\$$ or mask $\$)$ ).tw.

68. (crossover\$ or cross-over\$ or (cross adj1 over\$)).tw.

69. ( (allocat\$ or allot\$ or assign\$ or divid\$) adj3 (condition\$ or experiment\$ or intervention \$ or treatment $\$$ or therap\$ or control\$ or group\$)).tw.

70. or/58-69

71.57 and 70

72. limit 71 to $y r=“ 2004-2006 ”$

\section{Appendix 6. Current Contents search strategy}

lexp Videorecording/

2 exp Audiovisuals/

3 (video $\$$ or audiovisual\$).tw.

4 (interactive medi\$ or interactive multimedi\$).tw.

5 or/ $1-4$

6 exp "CONSENT (RESEARCH)"/ or exp CONSENT/

7 exp Truth Disclosure/

8 (truth adj disclosure).tw.

9 disclos $\$ . t w$.

10 consent.tw.

Audio-visual presentation of information for informed consent for participation in clinical trials (Review)

Copyright $\odot 2009$ The Cochrane Collaboration. Published by John Wiley \& Sons, Ltd. 
11 exp COMMUNICATION/

12 communicat\$.tw.

13 (inform\$ adj1 trial\$).tw.

14 exp Decision Making/

15 decision.mp. or decid\$.tw. [mp=title, subject heading word, abstract, instrumentation]

16 or/6-15

17 exp Clinical Trials/

18 (clinic $\$$ adj3 trial\$).tw.

19 ((vaccine\$ or drug\$) adj3 trial\$).tw.

20 exp Clinical Research/

21 exp Patient Selection/

22 (select\$ adj3 patient\$).tw.

23 exp Consumer Participation/

24 (participat\$ adj3 consumer\$).tw.

25 (participat $\$$ adj3 (client $\$$ or patient $\$$ )).tw.

26 exp Research Subjects/

27 (research\$ adj1 subject\$).tw.

28 (research\$ or experiment $\$$ or investigat $\$$ ).tw.

29 or/ $17-28$

305 and 16 and 29

31 randomi?ed controlled trial\$.af.

32 random\$.tw.

33 clinical trial.pt.

34 exp Clinical Trials/

35 (clin\$ adj25 trial\$).tw.

36 ((singl\$ or doubl\$ or treb\$ or tripl\$) adj25 (blind \$ or mask\$)).tw.

37 exp PLACEBOS/

38 placebo $\$ . t w$.

39 exp Study Design/

40 or/31-39

4130 and 40

42 (video\$ or audiovisual $\$$ ).tw. [mp=title, subject heading word, abstract, instrumentation]

43 (interactive and (media or medium or multimedia)).tw. [mp=title, subject heading word, abstract, instrumentation]

4442 or 43

45 consent.tw.

46 disclos\$.tw.

47 communicat\$.tw.

48 (inform\$ adj1 trial\$).tw.

49 (decision\$ or decid\$).tw. [mp=title, subject heading word, abstract, instrumentation]

50 or/ $45-49$

51 (clinic $\$$ adj3 trial\$).tw.

52 ((vaccin\$ or drug\$) adj3 trial\$).tw.

53 human experiment\$.tw.

54 (select\$ adj3 patient\$).tw.

55 (participat\$ adj3 patient\$).tw.

56 ((client\$ or consumer\$) adj3 participat\$).tw.

57 (research $\$$ adj1 subject $\$$ ).tw.

58 (research $\$$ or experiment $\$$ or investigat $\$$ ).tw.

59 or/ $51-58$

6044 and 50 and 59

61 randomized controlled trial\$.sh.

62 controlled clinical trial\$.sh.

63 random allocation.sh.

Audio-visual presentation of information for informed consent for participation in clinical trials (Review)

Copyright $\odot 2009$ The Cochrane Collaboration. Published by John Wiley \& Sons, Ltd. 
64 double blind method.sh.

65 single blind method.sh.

66 (clin\$ adj25 trial\$).ti,ab.

67 ( (singl\$ or doubl $\$$ or tripl $\$$ or trebl\$) adj25 (blind $\$$ or mask $\$)$ ).ti,ab.

68 placebo $\$$. sh.

69 placebo\$.ti,ab.

70 random $\$ . t i, a b$.

71 research design.sh.

72 or/ $61-71$

7360 and 72

\section{Appendix 7. CENTRAL (Cochrane Central Register of Controlled Trials) search strategy}

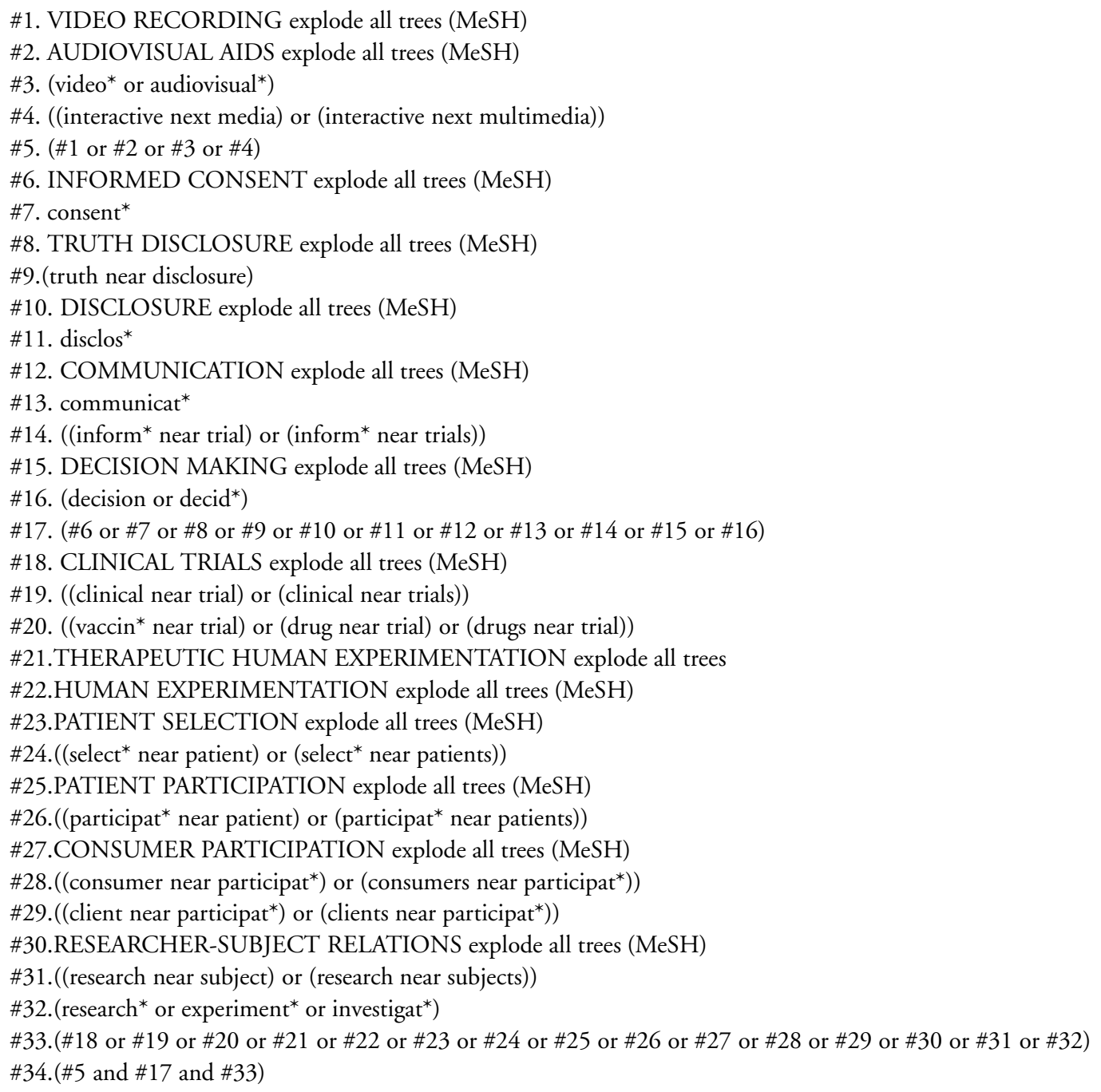

Audio-visual presentation of information for informed consent for participation in clinical trials (Review) 


\section{Appendix 8. ERIC search strategy}

(KW=(interactive and (media or medium or multimedia)) or KW=audiovisual* or KW=video*)

AND

(decision* or decide* or (inform* within 1 trial* $^{*}$ ) or communicat* or disclos* or consent)

AND

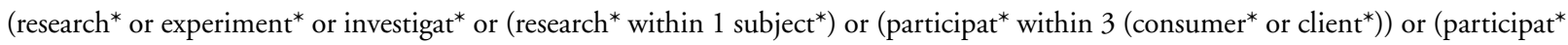
within 3 patient $\left.t^{*}\right)$ or $\left(\right.$ select* within 3 patient $\left.^{*}\right)$ or (human experiment*) or $\left(\left(\right.\right.$ vaccin $^{*}$ or drug* $)$ within 3 trial*) or $\left(\right.$ clinic $^{*}$ within3

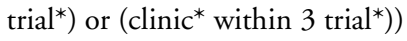

AND

$\left(\left(\left(\right.\right.\right.$ control$^{*}$ or clinical $\left.{ }^{*}\right)$ near trial $\left.{ }^{*}\right)$ or $\left(\left(\right.\right.$ singl $^{*}$ or doubl* or tripl* or trebl*) and $\left(\right.$ blind $^{*}$ or mask $\left.\left.{ }^{*}\right)\right)$ or $\left(\right.$ clin $^{*}$ near trial*) or random*)

\section{Appendix 9. Consumers \& Communicaton Group Specialised Register search strategy}

(video or "video recording")

AND

(S3 OR 'Informed consent" or cc.h)

\section{Appendix 10. Meta-analysis methods (retained for future updates)}

Analysis would include calculation of relative risk (RR) and 95\% confidence intervals (CI) for dichotomous outcomes and weighted mean difference (WMD) and 95\% CI for continuous outcomes, using Cochrane Review Manager (RevMan) software. The Chisquared test would be used to test for heterogeneity in outcomes. A fixed effects model would be used unless heterogeneity was found, in which case a random effects model would be used. Primary analyses would be on an 'intention to treat' basis.

Sensitivity analyses (to evaluate the effects of trial quality on meta-analysis results) and subgroup analyses (to assess the effects of the timing of the video intervention delivery relative to the informed consent process; the timing of outcome assessment with reference to the video intervention delivery; the use of the video intervention for the guardians of individuals asked to participate in a clinical study; and the effects on people with low literacy or people from other language groups) would be conducted if possible.

These methods will be retained for application in a future update of the review, if statistical pooling becomes possible.

\section{Appendix I I. Details of author/expert contact}

\begin{tabular}{|c|c|c|c|}
\hline Study ID/Expert Name & Contact person & Response? & Information obtained \\
\hline \multicolumn{4}{|l|}{ TRIAL AUTHORS } \\
\hline Agre 2003 & P. Agre & Yes & $\begin{array}{l}\text { Yes: confirmed that Mintz et al trial (cited in Agre et al 2003) } \\
\text { was published as Wirshing et al } 2005 \text {. Also provided contact } \\
\text { details to N. Kass, author of second queried trial cited in Agre } \\
\text { et al } 2003 \text {. Provided confirmation that Agre et al } 1994 \text { paper } \\
\text { was informed consent for therapeutic decision-making (and } \\
\text { excluded from review). Provided detailed information about } \\
\text { the Agre } 2003 \text { b study September } 122006\end{array}$ \\
\hline Benson 1988 & P.S. Appelbaum & Yes & Yes: provided details requested on author form. \\
\hline
\end{tabular}

Audio-visual presentation of information for informed consent for participation in clinical trials (Review) 
(Continued)

\begin{tabular}{|c|c|c|c|}
\hline Benson 1988 & P. Benson & No & - \\
\hline Dunn 2001 & L. Dunn & Yes & $\begin{array}{l}\text { Yes: confirmed that intervention did not contain audio-vi- } \\
\text { sual material; study excluded based on this information. Also } \\
\text { noted that an ongoing study using audio-visual intervention } \\
\text { is currently underway; recontacted to request details about } \\
\text { this study (whether it meets the review's selection criteria). } \\
\text { Author reply indicated that the ongoing study is about in- } \\
\text { formed consent for a hypothetical trial and so is not eligible } \\
\text { for this review }\end{array}$ \\
\hline Fureman 1997 & I. Fureman & No & - \\
\hline Hougham 2003 & G.Sachs & Yes. & $\begin{array}{l}\text { Yes: provided up to date contact details for G. Hougham; } \\
\text { also provided additional papers and contact details for several } \\
\text { researchers working in the field of informed consent }\end{array}$ \\
\hline Hougham 2003 & G. Hougham & Yes. & $\begin{array}{l}\text { Yes: initial response agreed to find information on trials con- } \\
\text { tained in paper. No further response }\end{array}$ \\
\hline Kass et al (cited in Agre 2003) & E. Emanuel & Yes & $\begin{array}{l}\text { Yes: J. Flory (first author) confirmed that the study by Kass et } \\
\text { al meets review inclusion criteria. Also confirmed additional } \\
\text { information was supplied by the first author (Nancy Kass) }\end{array}$ \\
\hline Kass et al (cited in Agre 2003) & N. Kass & Yes & $\begin{array}{l}\text { Yes: confirmed that study currently unpublished and study } \\
\text { details may be available at later date. Study to be included } \\
\text { in 'studies awaiting classification' as of August } 2007 \text { and re- } \\
\text { assessed at later date. Author provided further information } \\
\text { on the study as well as citation details (coauthors, title, insti- } \\
\text { tutional affiliation) }\end{array}$ \\
\hline Norris 1990 & Authors not traceable & & - \\
\hline Weston 1997 & J. Weston & Yes. & $\begin{array}{l}\text { Yes: confirmation of duplication of study (Weston 1995, ab- } \\
\text { stract); provision of all requested additional trial details }\end{array}$ \\
\hline Wirshing 2005 & D. Wirshing & Yes & $\begin{array}{l}\text { Yes: provided full text paper (just published) and additional } \\
\text { references }\end{array}$ \\
\hline \multicolumn{4}{|l|}{ EXPERTS } \\
\hline $\begin{array}{l}\text { J. Karlawish re informed con- } \\
\text { sent research (expert) }\end{array}$ & J. Karlawish & No & - \\
\hline $\begin{array}{l}\text { S. Kim re informed consent re- } \\
\text { search (expert) }\end{array}$ & S. Kim & Yes & $\begin{array}{l}\text { Yes: referred to other experts in research on interventions to } \\
\text { alter informed consent }\end{array}$ \\
\hline
\end{tabular}

Audio-visual presentation of information for informed consent for participation in clinical trials (Review) 
WHAT'S NEW

Last assessed as up-to-date: 7 November 2007.

\begin{tabular}{l|l|l}
\hline Date & Event & Description \\
\hline 9 April 2009 & Amended & Mittal 2007 added to excluded studies list. \\
\hline
\end{tabular}

\section{H I S T O R Y}

Protocol first published: Issue 3, 2002

Review first published: Issue 1, 2008

\begin{tabular}{lll}
\hline Date & Event & Description \\
\hline 19 March 2008 & Amended & Converted to new review format. \\
\hline 8 November 2007 & New citation required and conclusions have changed & Substantive amendment \\
\hline
\end{tabular}

\section{CONTRIBUTIONS OFAUTHORS}

KM and Caroline Crowther (see Acknowledgements) participated in the conceptualisation of the review and KM, Annie Brindley (see Acknowledgements) and Caroline Crowther prepared the protocol.

At review stage:

$\mathrm{RR}$ assisted in searching and selected studies for inclusion, extracted data, contacted authors, entered data into Revman, contributed to the review text and addressed feedback from editors and peer reviewers.

MP assessed studies for inclusion, extracted data, contributed to the review text and addressed feedback from editors and peer reviewers.

$\mathrm{KM}$ assisted in data extraction and contributed to the review text.

$\mathrm{SH}$ commented on the review and contributed to the review text.

\section{DECLARATIONSOF INTEREST}

None known 


\section{SOURCES OF SUPPORT}

\section{Internal sources}

- No sources of support supplied

\section{External sources}

- Department of Health and Ageing, Australia.

- Department of Human Services, Victoria, Australia.

\section{INDEX TERMS}

\section{Medical Subject Headings (MeSH)}

*Audiovisual Aids; ${ }^{*}$ Clinical Trials as Topic; *Informed Consent; *Patient Selection; Patient Education as Topic [ ${ }^{*}$ methods]; Randomized Controlled Trials as Topic

\section{MeSH check words}

Humans 